\title{
A unity of opposites in between Nrf1- and Nrf2-mediated responses to the endoplasmic reticulum stressor tunicamycin
}

\author{
Yu-ping Zhu ${ }^{1, \S}$, Shaofan $\mathrm{Hu}^{1, \S}$, Xufang $\mathrm{Ru}^{1, \S}$, Ze Zheng ${ }^{1}$, Zhuo Fan ${ }^{1}$, Lu Qiu ${ }^{1,2}$, and Yiguo Zhang ${ }^{1}$ * \\ ${ }^{1}$ The Laboratory of Cell Biochemistry and Topogenetic Regulation, College of Bioengineering and Faculty of Sciences, Chongqing \\ University, No. 174 Shazheng Street, Shapingba District, Chongqing 400044, China. \\ ${ }^{2}$ Present address: School of Life Sciences, Zhengzhou University, No. 100 Kexue Avenue, Zhengzhou 450001, Henan, China. \\ ${ }^{\S}$ Contributed equally to this work. \\ *Correspondence should be addressed to Yiguo Zhang (Email: yiguozhang@cqu.edu.cn, or eaglezhang64@gmail.com)
}

Abstract: The water-soluble Nrf2 is accepted as a master regulator of antioxidant responses to cellular stress, it was also identified as a direct target of the endoplasmic reticulum (ER)-anchored PERK. However, the membrane-bound Nrf1 response to ER stress remains elusive. Herein, we report a unity of opposites in both Nrf1- and Nrf2-coordinated responses to the ER stressor tunicamycin (TU). The TU-inducible transcription of Nrf1 and Nrf2, as well as GCLM and HO-1, was accompanied by activation of ER stress signaling networks. The unfolded protein response (UPR) mediated by ATF6, IRE1 and PERK was significantly suppressed by Nrf1 $\alpha$-specific knockout, but hyper-expression of Nrf2, GCLM and HO-1 was retained in Nrf1 $\alpha^{-/-}$cells. By contrast, Nrf2 $2^{-/-\Delta T A}$ cells with a genomic deletion of its transactivation domain resulted in significant decreases of GCLM, HO-1 and Nrf1; this was accompanied by partial decreases of IRE1 and ATF6, but not PERK, along with an obvious increase of ATF4. Notably, Nrf1 glycosylation and its trans-activity to mediate transcriptional expression of $26 \mathrm{~S}$ proteasomal subunits were repressed by TU. This inhibitory effect was enhanced by $N r f 1 \alpha^{-1-}$ and $N r f 2^{-1-\Delta T A}$, but not by a constitutive activator caNrf2 ${ }^{\Delta N}$ (that increased abundances of non-glycosylated and processed Nrf1). Furthermore, caNrf2 $2^{\Delta N}$ also enhanced induction of PERK and IRE1 by TU, but reduced expression of ATF4 and HO-1. Such distinct roles of Nrf1 and Nrf2 are unified to maintain cell homeostasis by a series of coordinated ER-to-nuclear signaling responses to TU. Overall, Nrf1 $\alpha$ acts in a cell-autonomous manner to determine transcription of most of UPR-target genes, albeit Nrf2 is also partially involved in this process.

Keywords: Nrf1, Nrf2, ER stress, unfolded protein response, redox signaling, tunicamycin, proteasome, glycosylation

\section{Introduction}

As a highly-evolved organelle of eukaryotic cells, endoplasmic reticulum (ER) is of crucial importance to be involved in biosynthesis of secretory and membrane proteins, as well as lipids including cholesterol, proper folding of proteins and specific post-translational modifications of those proteins sorted out of the ER to their destination organelles. The ability of the ER to orchestrate intracellular proteins and lipids is also severely challenged by a vast variety of physio-pathological stresses and environmental stimuli [1]. Consequently, disruption of such function of ER leads to accumulation of a large amount of unfolded and/or misfolded proteins in the lumen of this organelle, destroying the original homeostasis of the cell; this is known as ER stress to activate the unfolded protein response (UPR) [2]. In metazoans, the UPR is mediated by three major axes of signaling pathways from: i) PERK (PRKR-like ER kinase)-elF2 $\alpha$ (eukaryotic translation initiation factor $2 \alpha$ ) to ATF4 and Chop; ii) IRE1 (inositol-requiring protein 1) to XBP1 (X-box binding protein 1); and iii) ATF6 [3]. In early stages of UPR, those unfolded proteins are allowed for binding to an ER luminal-resident chaperone BIP (immunoglobulin heavy chain-binding protein homolog, also called glucose regulated protein 78, i.e. GRP78) [4]. This critical event results in the induction of those ER-associated sensors PERK, IRE1 and ATF6, so that their relevant UPR signaling pathways are subsequently activated [5] (as illustrated in Figure 1).

Upon activation of UPR, it is also accompanied by concomitant and secondary induction of a complex signaling networks, which include pro-survival mechanisms involving antioxidant responses, ER-associated degradation (ERAD) ER biogenesis and autophagy [6]. Notably, the potential role of UPR in maintaining redox homeostasis is attracting 


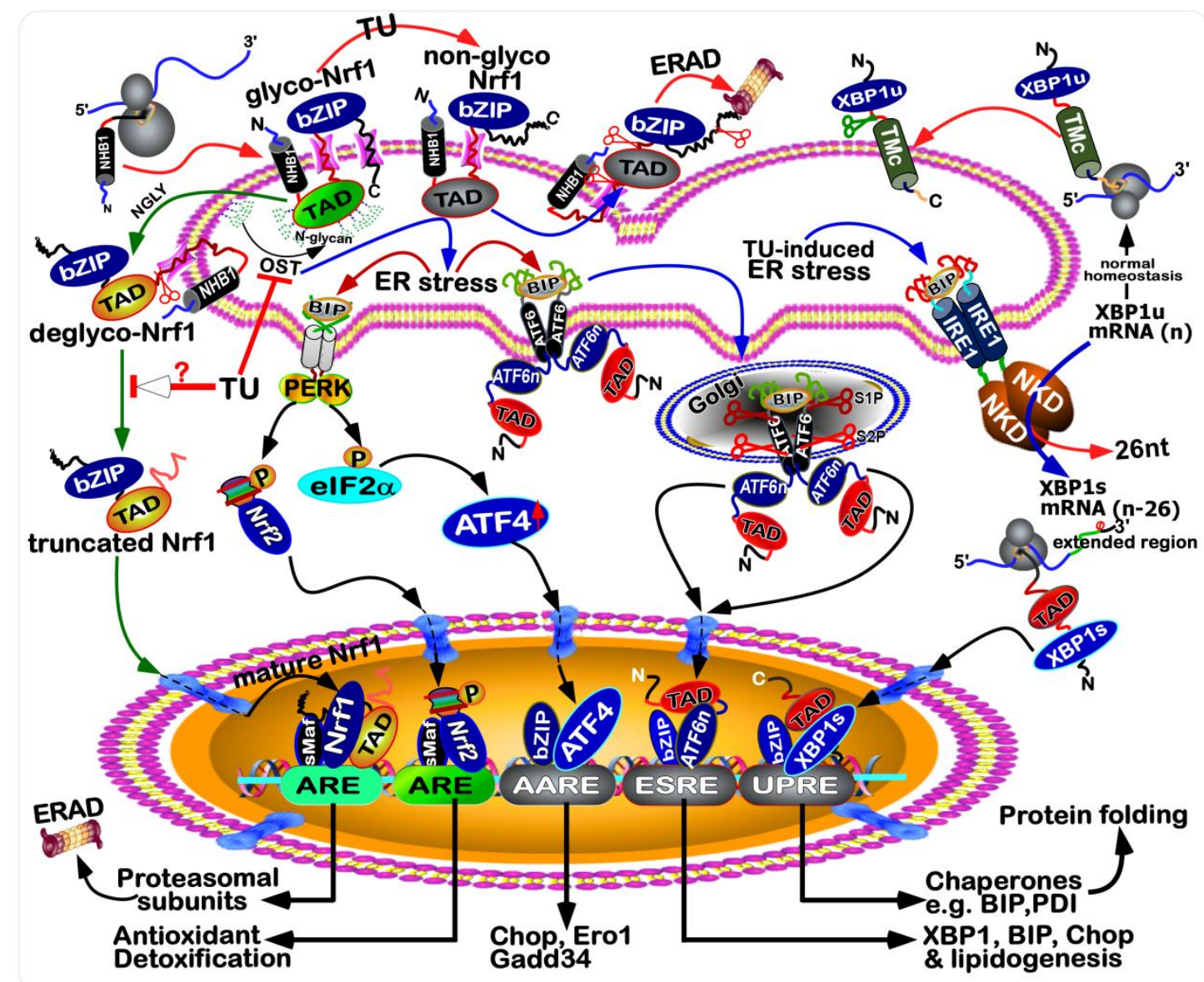

Figure 1. Inhibition of Nrf1 N-glycosylation by TU in the ER-to-nuclear signaling responses.

A model is herein proposed to explain the inhibition of $\mathrm{Nrf1} \mathrm{N}$-glycosylation by $\mathrm{TU}$, as a classic stressor, in order to block its deglycosylation and proteolytic processing to yield a mature CNC-bZIP factor. The resulting non-glycosylated Nrf1 and others are subject to the ER-associated degradation (ERAD) pathway. If the non-glycosylated and unfolded proteins are accumulated so much as to stimulate ER stress. Thereby, inhibition of Nrf1 by TU is also accompanied by activation of canonic ER-to-nuclear response signaling mediated by PERK, IRE1 and ATF6, as reviewed by the authors [1-4]. Of note, PERK was also identified as a direct upstream kinase of Nrf2, as well as elF2 $\alpha$ [10], albeit this CNC-bZIP protein has been well-characterized as a master antioxidant transcription factor to regulate ARE-driven genes. In this response, ATF4 is selectively translated by the elF2 $\alpha$-control machinery to regulate target genes (including Chop, Ero1, Gadd34) by their amino acid response elements (AARE) in the promoter regions. To get rid of ER stress, ATF6 was activated by consecutive proteolytic processing of this bZIP protein by Site-1 and Site-2 proteases (i.e., S1P and S2P). The full-length mRNA of XBP is alternatively spliced to remove its 26 nucleotides at nearly $3^{\prime}$-end, such that the subsequent portion of its open reading frame is shifted to yield a longer inducible protein isoform called XBP-1s than its original protein of XBP-1u. Consequently, distinct subsets of cognate target genes containing either cis-regulatory ESRE (ER stress response element) or UPRE (unfolded protein response element) are regulated transcriptionally by ATF6n (i.e., its active N-terminal portion) and XBP-1s, respectively.

great interest of workers in different fields [7]. Clearly, it is generally accepted that redox responses are mediated by two important antioxidant transcription factors Nrf1 [i.e. nuclear factor erythroid 2 (NF-E2) p45-related factor 1, also called Nfe2I1] and Nrf2 [8]. These two members of the cap'n'collar (CNC) basic-region leucine zipper (bZIP) family can predominantly regulate transcription of antioxidant response element (ARE)-driven genes involved in detoxification 
and other cytoprotective adaptations. Besides, Nrf2 was also previously reported to be significantly up-regulated by amyloid beta-induced ER stress leading to UPR [9]. As one of the three UPR transducers, PERK had been identified to be a direct upstream kinase of Nrf2 [10], which is well characterized as a master antioxidant transcription factor to counterbalance the harmful effects of reactive oxygen species (ROS) on cells. Under ER stress, Nrf2 is phosphorylated by PERK, such that the phosphorylated Nrf2 dissociates from its negative regulator KEAP1 and then translocated the nucleus leading to transactivation of ARE-battery genes $[11,12]$. In the meanwhile, elF2 $\alpha$ is also phosphorylated by PERK, thereby repressing general protein translation, but promoting selective protein translation of ATF4 [13-15]. Furtherly, the heterodimer consisting of ATF4 and Nrf2 binds to the stress-response element of heme oxidase-1 (HO-1), before inducing this gene expression [16]. Collectively, these demonstrate that Nrf2-mediated expression of ARE genes is activated in the UPR signaling to ER stress. However, whether (and how) the ER membrane-associated Nrf1-mediated response signaling is triggered to the accompaniment of UPR remains elusive, to date.

Although the ER-associated Nrf1 adapts a unique membrane-topology (as shown in Figure 1), at least in part of which is similar to the consensus transmembrane topology of PERK, IRE1 and ATF6. Thereby, it is postulated that the membrane-bound Nrf1 should, theoretically, has a strong capacity of being induced by ER stress, as described for the homologue of Caenorhabditis elegans Skn-1 [17-19]. Intriguingly, ectopic Nrf1 appeared to be de facto not activated by each of UPR signaling pathways, but conversely, activation of Nrf1 by tert- butylhydroquinone (tBHQ) or arsenic to up-regulate ARE-driven genes responsible for antioxidant, detoxification and cytoprotection was repressed by classic ER stressors, such as tunicamycin (TU), thapsigargin (TG) and brefeldin A (BFA) [20, 21]. As such, the electrophoretic mobility of Nrf1 and its subcellular redistribution were altered by TU and BFA, but not TG [21]. This suggests that Nrf1 is modified in an ER stress-stimulated post-translational fashion (i.e. N-glycosylation, deglycosylation, ubiquitination, and proteolysis). By sharp contrast, the water-soluble Nrf2 was identified as a direct target of PERK triggered by TU, insofar as to activate antioxidant and detoxification responses [10, 12].

It is important to note a unique UPR-independent mechanism whereby the transactivation activity of Nrf1, rather than Nrf2, to up-regulate transcription of all those genes encoding 26S proteasomal (PSM) subunits is induced by low concentrations of its inhibitors [22-24]. Such being the case, proteasomal inhibition can also result in accumulation of oxidative ubiquitinated proteins, thereby triggering ER stress [24-26]. In fact, ER stress-inducible UPR signaling pathways were endogenously activated by loss of mouse Nrf1 function in the homozygous (Nrf1 $1^{-1}$ ) hepatocytes [27]. Contrarily, both similar ER stress responses and steatosis were enhanced by half loss of Nrf1 in the heterozygous $\left(\mathrm{Nrf1}^{+/-}\right)$livers, when compared with wild-type $\left(\mathrm{Nrf1}^{+/+}\right)$livers, in response to 26S proteasomal inhibition [27]. Such concurrence of severe oxidative stress with UPR in mouse $\mathrm{Nrf1}^{-1-}$ livers results from down-regulation of antioxidant, detoxification and proteasomal genes. In the proteasomal compensatory response to limited extents of proteasome inhibitors [28], Nrf1 is subjected to the multistep processing of Nrf1 and its nuclear translocation [29], albeit the inhibition of proteasome-mediated ERAD is also one of the causes of ER stress [24, 26, 30]. These facts demonstrate that Nrf1 possesses an essential cytoprotective role against development of hepatosteatosis by maintaining the ER homeostasis, but the underlying mechanism remains unknown.

To unveil the mystery players of Nrf1 and Nrf2 in UPR, it is necessary to gain insights into distinct roles of both CNC-bZIP factors in the ER response to TU. Herein, we report a unity of opposite roles of Nrf1 and Nrf2 in the cellular responses to treatment of TU in different genotypic cell lines. TU-induced UPR signaling by ATF6, IRE1 and PERK was 
significantly suppressed by Nrf1 $\alpha$-specific knockout, but Nrf2, GCLM and HO-1 was highly expressed in Nrf1 $\alpha^{-1-}$ cells. By contrast, $N r f 2^{-/-\Delta T A}$ cells with a genomic deletion of its transactivation domain resulted in significant decreases of GCLM, HO-1 and Nrf1. This was also accompanied by partial decreases of IRE1 and ATF6, but not PERK, along with an obvious increase of ATF4. Notably, glycosylation of Nrf1 and its ability to transactivate expression of 26S proteasomal subunits were markedly repressed by TU. This inhibitory effect was enhanced by $N r f 1 \alpha^{-/-}$and $N r f 2^{-1-\Delta T A}$, but not by a constitutive activator caNrf2 $2^{\Delta N}$ because of increased abundances of non-glycosylated and processed Nrf1. In addition, $c a N r f 2^{\Delta N}$ enhanced induction of PERK and IRE1 by TU, but reduced ATF4 and HO-1. Collectively, such distinctive roles of Nrf1 and Nrf2 in the ER-to-nuclear signaling responses to TU are unified for maintaining cell homeostasis. Overall, our results demonstrate that Nrf1 $\alpha$ acts as a dominant player in a cell-autonomous manner to regulate most of UPR gene expression, while Nrf2 is partially involved in this process by IRE1, at least in this experimental setting.

\section{Results}

Nrf1 $\alpha$ and Nrf2 contribute to differential expression of responsive genes to ER stress in four different cell lines.

To explore distinct functions of Nrf1 and Nrf2 in the putative ER stress response, herein we employed four different genotypic cell lines, which had been established by gene-editing with the presence or absence of Nrf1 $\alpha$ and Nrf2 [31]. Consistently, these selected cell lines were re-identified by Western blotting of Nrf1 and Nrf2before RNA sequencing. As shown in Figure 2A, wild-type HepG2 cell line $\left(\mathrm{Nrf1} / 2^{+/+}\right)$served as a control for the expression of Nrf1 and Nrf2. By contrast, Nrf1 $\alpha^{-1-}$ cell line exhibited a clear disappearance of the intact full-length Nrf1 $\alpha /$ TCF11 and its derivatives (which are embodied by glycoprotein-A, deglycoprotein-B and N-terminally-truncated C-/D-forms of between 140and 120-kDa; their detailed identifications had been described in our previous work [24, 32]). However, expression of shorter Nrf1-truncated E-form appeared to be enhanced in $\mathrm{Nrf1} \alpha^{-/-}$cell with unaltered F-form, when compared with $\mathrm{Nrf1} / 2^{+/+}$cells (Figure 2A, upper panel). This implies a potential molecular compensatory mechanism, because E- and F-isoforms of Nrf1 may be generated by translation of a shorter-length open reading frame of mRNA resulting from the alternative first exon (i.e. $\mathrm{Nrf1}^{\Delta \mathrm{N}}$ ) $[8,24]$, in addition to proteolytic processing of longer Nrf1 isoforms. Intriguingly, almost no expression of the Nrf1-truncated E-isoform was determined in both cell lines of Nrf2 ${ }^{-1-\Delta T A}$ and $\operatorname{caNrf2}{ }^{\Delta N}$, but they gave a modest decrease in abundance of Nrf1 F-form. Similarly, abundances of Nrf1 $\beta$ bands close to 70-kDa appeared to be unaffected by knockout of $\mathrm{Nrf1} \alpha$, but was significantly augmented by $\mathrm{Nrf} 2^{-/-\Delta T A}$ and rather reduced by $c a N r f 2^{\Delta N}$ (Figure 2A, upper panel). These data suggest a potential effect of $\mathrm{Nrf2}$ on alternative translation of either $\mathrm{Nrf1}^{\Delta \mathrm{N}}$ or Nrf1 $\beta$, but another possible role of Nrf2 in alternative transcription of Nrf1 cannot also be ruled out, albeit detailed mechanism(s) remains unknown.

By contrast with $\mathrm{Nrf1} / 2^{+/+}$cells, $\mathrm{Nrf1} \alpha^{-/-}$cells gave rise to a dramatic increase in the expression of Nrf2 between 100- and 110-kDa (Figure 2A, middle panel); such a demonstrating effect of Nrf1 on the expression of Nrf2 was also described previously [31]. Nonetheless, similar longer Nrf2 between $\sim 100$ - and 110-kDa were completely abolished in $\mathrm{Nrf2}^{-\mathrm{I}_{\mathrm{ATA}}}$ cells but replaced by additional smaller molecular weight polypeptides with a genomic deletion of its transactivation domain. Notably, the intact full-length Nrf2 of 110-kDa was also totally abolished by caNrf2 ${ }^{\Delta N}$, but it retained a major short $\mathrm{Nrf2}$ of $\sim 100-\mathrm{kDa}$, together with a few of minor small polypeptides (Figure $2 \mathrm{~A}$, middle panel). These suggest a possible proteolytic processing of Nrf2 within its N-terminal Neh2 domain (which is highly conserved with the Neh2L region immediately adjacent to the N-terminal domain of Nrf1). 

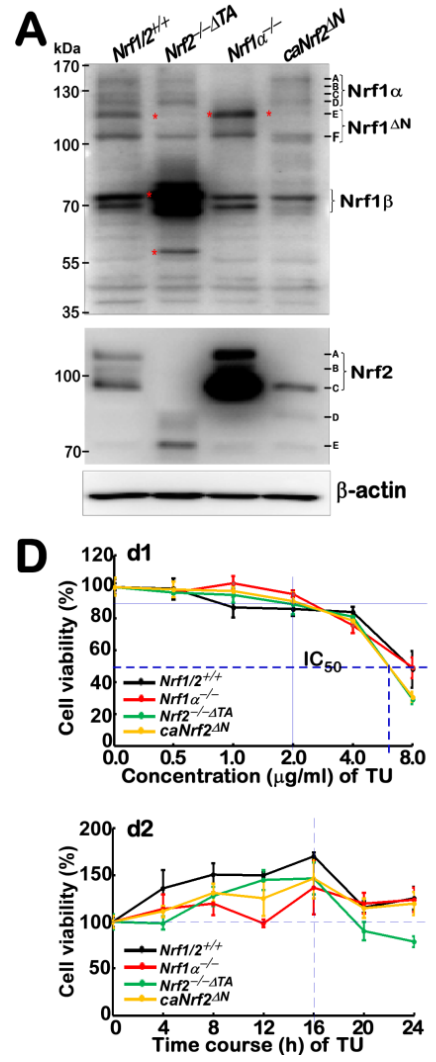
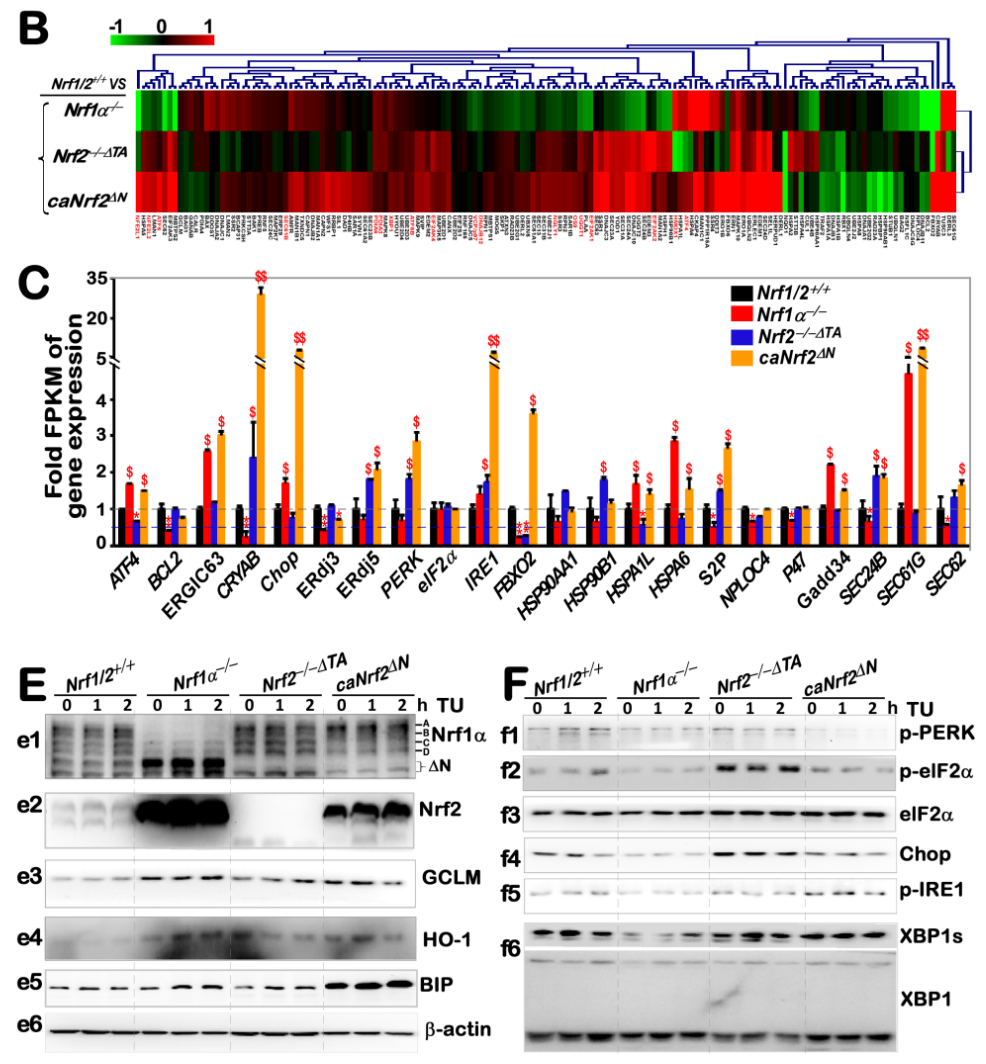

Figure 2. Distinct contributions of Nrf1 $\alpha$ and Nrf2 to differential expression of ER stress-related genes.

(A) Distinct protein levels of Nrf1 and Nrf2 in different genotypic cell lines $N r f 1 / 2^{+1+}, N r f 1 \alpha^{-1-}, N r f 2^{-1-\Delta T A}$ and $c a N r f 2^{\Delta N}$ were determined by Western blotting with specific antibodies. (B) A heatmap was made by the Log2-based RPKM values, representing differential expression profiles of ER stress-related genes by comparison to those obtained from $\mathrm{Nrf1}^{+/+}$cells. Different changes in the basal expression of these genes are shown to distinct degrees of colors. (C) The expression of ER stress responsive genes was also evaluated by relative RPKM values. Significant statistical decreases were indicated by ${ }^{*} p<0.01$ or ${ }^{* *} p<0.001$, whereas significant increases were represented by $\$, p<0.01$ or $\$ \$, p<0.001$. (D) The viability of these cell lines that had been treated with different concentrations of TU for $24 \mathrm{~h}(d 1)$, or treated with $2 \mu \mathrm{g} / \mathrm{ml}$ of TU for different lengths of time (d2), were determined by an MTT-based assay. (E) The protein levels of antioxidant genes (i.e. $e 1$ to $e 6$ ) and (F) the ER stress-related genes (i.e. $f 1$ to $f 6$ ) in the experimental cells, that had been treated with $2 \mu \mathrm{g} / \mathrm{ml}$ of TU for a short time (from 0 to $2 \mathrm{~h}$ ), were examined by Western blotting with indicated different antibodies.

Subsequently, the fidelity of total RNAs purified from the above-identified four cell lines was rigidly confirmed to be available for RNA-sequencing. A heatmap of the sequencing data revealed 157 of differentially expressed genes clustered responsibly for ER stress in $\mathrm{Nrf1} \alpha^{-1-}, \mathrm{Nrf2} 2^{-1-\Delta T A}$ or caNrf2 $2^{\Delta N}$ by comparison with $\mathrm{Nrf1} / 2^{+/+}$cells (Figure 2B). Amongst them, relative highly expressed genes were differed in these four cell lines as shown graphically (Figure 2C). Further analysis of these data, taken together with the aforementioned alternations in abundances of Nrf1 and Nrf2, suggests that basal expression of 7 genes encoding ATF4, Chop, Gadd34, ERGIC63, HSPA1L, HSPA6 and Sec61 $\gamma$ could be regulated predominantly by Nrf2, because their mRNA levels were significantly increased in $N r f 1 \alpha^{-1-}$ and caNrf2 ${ }^{4 N}$ cells, but obviously diminished or abolished in $\mathrm{Nrf}^{-1-\Delta T A}$ cells (Figure $2 \mathrm{C}$ ). By contrast, Nrf1 $\alpha / \mathrm{TCF} 11$ may be primarily involved in regulating basal expression of another 9 genes encoding BCL2, CRYAB, ERdj3, PERK, FBXO2, S2P, NPLOC4, $\operatorname{Sec} 24 \beta$, and Sec62. This is due to the fact that their basal mRNA abundances were markedly repressed in $\mathrm{Nrf}_{1} \alpha^{-1-}$ 
cells, albeit with high expression of Nrf2. Conversely, a few of these genes regulated by Nrf1 $\alpha$ might also be inhibited by Nrf2, because their mRNA expression levels were strikingly recovered by $N r f 2^{-/-\Delta T A}$ (or caNrf2 $2^{\Delta N}$ with a constitutive deletion of cytoplasmic Keap1-binding Neh2 domain of Nrf2, with an unidentified nuclear function of this domain). Notably, IRE1 could be co-regulated by both Nrf1 $\alpha$ and Nrf2, because its mRNA expression levels were unaffected by knockout of Nrf1 or Nrf2, but significantly elevated by caNrf2 $2^{\Delta N}$, implying a possible release of inhibition by Neh2.

Distinct effects of Nrf1 $\alpha$ and Nrf2 in protein expression of responsive genes to early TU-induced ER stress.

To determine effects of Nrf1 and Nrf2 on differential expression of putative genes in response to the ER stressor TU, we performed Western blotting to examine changes in the protein levels of early TU-responsive genes expressed, for a short period of time, in the aforementioned four cell lines with the presence or absence of Nrf1 $\alpha$ and Nrf2. Firstly, cytotoxity of TU was evaluated to provide an optimal concentration of this chemical that was treated for an optimal time course (Figure 2D). A half of maximal inhibitory concentration (IC 50 ) of TU treated in $N r f 2^{-1-\Delta T A}$ or caNrf2 $2^{\Delta N}$ cells was $\sim 6.5 \mu \mathrm{g} / \mathrm{ml}$, while another $\mathrm{IC}_{50}$ of TU treatment of $N r f 1 \alpha^{-/-}$or $N r f 1 / 2^{+/+}$cells was close to $8.0 \mu \mathrm{g} / \mathrm{ml}$ (Figure 2D1). Of note, $2.0 \mu \mathrm{g} / \mathrm{ml}$ of TU, with almost no obvious cytotoxity for $24 \mathrm{~h}$ (Figure 2D2), was selected for the use of our subsequent experiments.

Western blotting revealed a modest increase in abundances of longer inactive Nrf1 isoform-A/B was examined following 2-h TU-treatment of $N r f 1 / 2^{+/+}$or caNrf2 ${ }^{\Delta N}$, but not $N r f 2^{-/-\Delta T A}$ cells (Figure 2E1); this appeared consistent with our previous work [24, 33]. As such, two shorter active isoforms-C/D of Nrf1 was enhanced by TU treatment of $\mathrm{Nrf2} 2^{-1-\Delta T A}$ cells only. Notably, either $\mathrm{Nrf2}$ or caNrf2 ${ }^{\Delta \mathrm{N}}$ protein levels were promoted by TU treatment of $N r f 1 \alpha^{-/-}$or $c a N r f 2^{\Delta N}$ cells, respectively (Figure 2E2). Further examination unraveled that the protein expression of ARE-driven genes encoding GCLM and HO-1 (regulated Nrf1 and/or Nrf2) was significantly increased by TU treatment of $\mathrm{Nrf1}^{-/-}$ and caNrf2 $2^{\Delta N}$ cells, when compared with $\mathrm{Nrf1} / 2^{+/+}$cells (Figure $2 \mathrm{E} 3$ and 2E4). Conversely, this antioxidant effect was partially reduced in TU-treated $\mathrm{Nrf2}^{-1-\Delta T A}$ cells. Taken altogether, these data demonstrate that short-term stimulation of TU can also trigger induction of antioxidant gene response mediated by Nrf1 and Nrf2.

Furtherly, distinct time-dependant increases in the chaperone BIP (also called GRP78, as a landscape signature of classic TU-induced ER stress response) were determined following TU treatment of caNrf2 ${ }^{\Delta N}, N r f 1 \alpha^{-1-}, N r f 2^{-1-\Delta T A}$ or $\mathrm{Nrf1} / 2^{+/+}$cells, when compared with their untreated controls (Figure 2E5). Of note, higher expression levels of basal and TU-stimulated BIP proteins was found in caNrf2 $2^{\Delta N}$ cells. By contrast, significant elevation of Nrf2 in Nrf1 $\alpha^{-1-}$ cells only gave rise to a considerable level of BIP, but was only slightly suppressed by knockout of Nrf2 (in Nrf2 ${ }^{-/-\Delta T A}$ cells) to a similar level to that obtained from $\mathrm{Nrf1} / 2^{+/+}$cells. Collectively, these data indicate that both Nrf1 and Nrf2 are required for BIP expression in a short-term ER stress response to TU. Western blotting examination of the PERKelF2 $\alpha /$ Nrf2 -Chop pathway revealed that both phosphorylated PERK and elF2 $\alpha$ proteins, along with total Chop were stimulated by TU treatment in $\mathrm{Nrf1} / 2^{++}$cells (Figure 2F1, 2F2 and 2F4). Interestingly, their expression was obviously

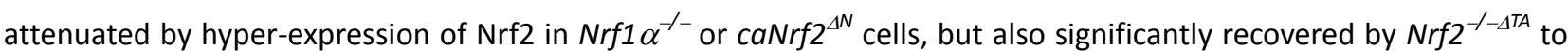
considerable high levels relative to those obtained from $\mathrm{Nrf1} / 2^{+/+}$cells; such alternations appears to be independent of stimulation by TU. No matter what it is, these observations demonstrate that Nrf1 and Nrf2 have exerted opposing roles for the PERK-elF2 $\alpha$-Chop signaling response to a short-term stimulation of TU. Further examination of the IRE1-XBP1 pathway unraveled that basal and TU-stimulated expression of $\mathrm{p}$-IRE and XBP1s appeared to be reduced in $\mathrm{Nrf1} \alpha^{-1-}$ cells (Figure 2F5 and 2F6), but p-IRE1 expression was enhanced in caNrf2 ${ }^{\Delta N}$ cells, albeit no changes of both 
proteins were observed in $N r f 2^{-/-\Delta T A}$ by comparison with equivalents of $N r f 1 / 2^{+/+}$cells. Overall, these results indicate that Nrf1 $\alpha$, but not Nrf2, is required for expression of auto-phosphorylated IRE1 and its target product XBP1s.

Nrf1 $\alpha$ and Nrf2 mediate distinct transcriptional responses to the long-term TU-stimulated ER stress.

The above experiments showed no changes in protein expression levels of some responsive genes at the early stages of TU-induced ER stress (Figure 2E,2F). Particularly, a few of them were de facto auto-activated in a TU-independent manner under the untreated homeostatic conditions. Thereby, the TU-treated time was further extended from $4 \mathrm{~h}$ to $24 \mathrm{~h}$, in order to determine distinctions in between Nrf1- and Nrf2-mediated transcriptional responses to a long-term TU-stimulated ER stress. For this, four different genotypic cell lines had been treated with TU for distinct lengths of time from 0 to $24 \mathrm{~h}$, before these genotypic mRNAs were subjected to real-time quantitative PCR (qRT-PCR) analysis. The results demonstrated that TU treatment of $\mathrm{Nrf1} / 2^{+/+}$cells triggered time-dependent increases in transcriptional expression of Nrf1 and Nrf2 (Figure 3A, 3B). By sharp contrast, both basal and TU-stimulated mRNA expression levels of $N r f 1$ were substantially abolished by $N r f 1 \alpha^{-/-}$and also significantly diminished by $N r f 2^{-/-\Delta T A}$, but not by caNrf2 ${ }^{\Delta N}$ (Figure 3A). This further supports our previous notion that the Nrf1 gene transcription is regulated positively by itself factor Nrf1 $\alpha$ (and derivatives) together with Nrf2, as described by Qiu et al [31]. Conversely, basal mRNA expression of $\mathrm{Nrf2}$ was elevated in $\mathrm{Nrf1} \alpha^{-1-}$ cells (with high abundances of Nrf2) to a 3.5-fold extent, as compared to the control value of $\mathrm{Nrf1} / 2^{+/+}$cells (Figure 3B). TU-treated $\mathrm{Nrf1} \alpha^{-/-}$cells only gave rise to a modest increase in expression of $\mathrm{Nrf2}$ mRNA following treatment for $8 \mathrm{~h}$ to $16 \mathrm{~h}$, but subsequently, this increased expression was significantly suppressed to a lower level than its basal value. However, basal and TU-inducible mRNA expression levels of Nrf2 were enhanced by $\operatorname{caNrf2} 2^{\Delta N}$ with no decreases in its induction, but all completely abolished by $N r f 2^{-1-\Delta T A}$ (Figure 3B). These collective results indicate that transcription of $\mathrm{Nrf2}$ gene is regulated positively by itself Nrf2 factor, but also negatively (at least in part) by Nrf1 and/or its downstream factors.

Further qRT-PCR analysis of $\mathrm{Nrf1} / 2^{+/+}$cells revealed that a TU-stimulated increase in the mRNA expression of BIP (as a classic marker of ER stress-induced UPR) from $8 \mathrm{~h}$ to $24 \mathrm{~h}$ following treatment, which occurred with a peak of TU induction at $12 \mathrm{~h}$ (Figure $3 \mathrm{C}$ ). By contrast, such TU-inducible mRNA expression of BIP was substantially suppressed and postponed in $\mathrm{Nrf1} \alpha^{-/-}$cells (with hyper-expression of Nrf2). Also, $\mathrm{Nrf2} 2^{-1-\Delta T A}$ cells only gave a modest reduction of TU-inducible BIP expression, with a lowered peak at $12 \mathrm{~h}$ and a subsequent downward course to $24 \mathrm{~h}$ after treatment. Collectively, these indicate that Nrf1 is required for transcriptional regulation of UPR-target BIP gene, while Nrf2 is partially involved in this response to TU. However, caNrf2 $2^{\Delta N}$ (acting as a constitutive CNC-bZIP activator) also caused a partial decrease of TU-inducible BIP expression (Figure 3C). This decrease is attributable to a deletion of the Keap1binding Neh2 domain from Nrf2 (to yield $c a N r f 2^{\Delta N}$ ), albeit the nuclear function of Neh2 is not yet identified to date.

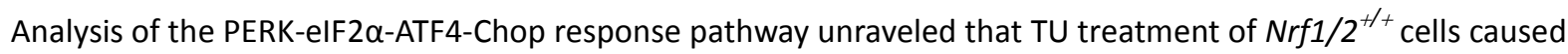
distinct time-dependent increases in mRNA levels of PERK, ATF4 and Chop from $8 \mathrm{~h}$ to $24 \mathrm{~h}$ (Figure 3D,3E,3F), which occurred with their respective peaks at $8 \mathrm{~h}, 24 \mathrm{~h}$ and $16 \mathrm{~h}$. Of note, TU-inducible expression of PERK was substantially prevented in $\mathrm{Nrf1} \alpha^{-/-}$cells (with high expression of Nrf2), but appeared to be almost unaffected by $\mathrm{Nrf2} 2^{-/-\Delta T A}$, when compared with those obtained from Nrf1/2 $2^{+/+}$cells (Figure 3D). This suggests that Nrf1 $\alpha$, but not Nrf2, is essential for transcriptional regulation of $P E R K$ in response to TU. Nevertheless, caNrf2 ${ }^{\Delta N}$ gave rise to rather significant increments in basal and TU-induced mRNA expression of PERK (Figure 3D). Hence, this implies that, once Nrf2 is localized in the nucleus, its Neh2 domain might serve as a putative dominant trans-repressor of the PERK gene. Known as a direct 
substrate of PERK, elF2 $\alpha$ is phosphorylated in the ER response to TU [4] and then contributes to selective translation of ATF4 (Figure 1). Herein, qRT-PCR showed that a time-dependent increment in ATF4 mRNA levels was induced in $\mathrm{Nrf1} / 2^{+1+}$ cells that had been treated for $8 \mathrm{~h}$ to $24 \mathrm{~h}$ with TU, which was peaked at $24 \mathrm{~h}$ (Figure 3E). Such late stages of TU-inducible ATF4 response after $16-\mathrm{h}$ treatment were blocked in $N r f 1 \alpha^{-/-}$or caNrf2 ${ }^{\Delta N}$ cells. Conversely, Nrf2 ${ }^{--\Delta T A}$ led to a remarkable accelerated promotion of ATF4 expression induced by TU, with an early peak at $12 \mathrm{~h}$ (Figure $3 \mathrm{E}$ ). These results indicate that Nrf2, but not $\mathrm{Nrf1} \alpha$, acts as a dominant trans-repressor of ATF4 gene in cellular response to TU. Further qRT-PCR analysis of Chop (as a downstream target gene of ATF4) revealed that its TU-inducible mRNA expression was markedly blocked by $\mathrm{Nrf1} \alpha^{-/-}$, and also partially inhibited by $\mathrm{Nrf2} 2^{-/-\Delta T A}$, but seemed to be unaffected by caNrf2 ${ }^{\Delta N}$ (Figure 3F). Thereby, it is inferable that Nrf1 $\alpha$ contributes primarily to transcriptional regulation of Chop gene, albeit Nrf2 is partially involved in this process.
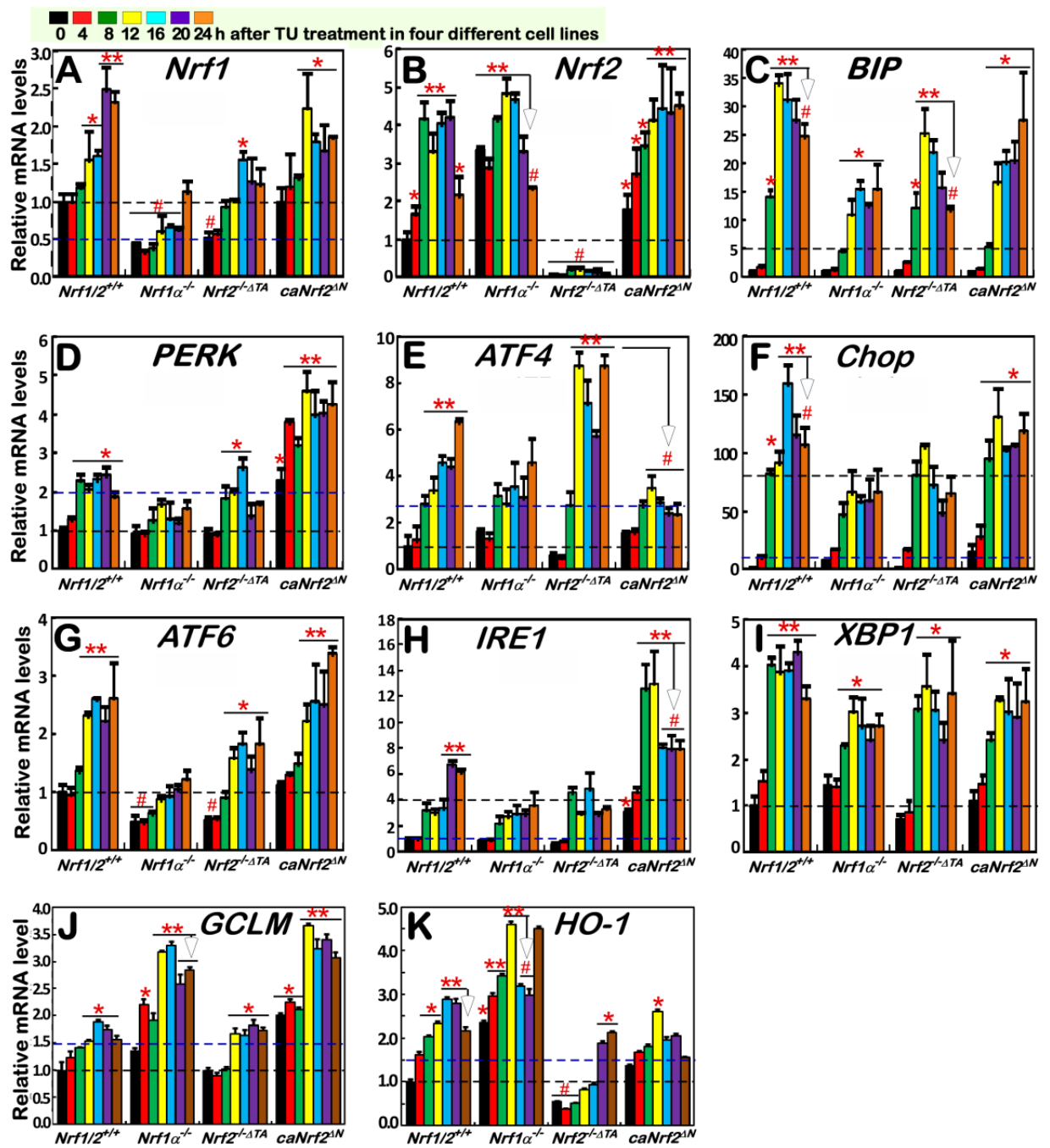

Figure 3. TU-inducible changes in the mRNA expression of distinct responsive genes.

Distinct cell lines of $N r f 1 / 2^{+/+}, N r f 1 \alpha^{-1-}, N r f 2^{-1-\Delta T A}$ and $c a N r f 2^{\Delta N}$ were treated with $2 \mu \mathrm{g} / \mathrm{ml}$ TU for the indicated times from 0 to $24 \mathrm{~h}$. Then, TU-inducible mRNA expression of distinct responsive genes was determined by real-time qPCR. These genes include Nrf1 (A), Nrf2 (B), BIP/GRP78 (C), PERK (D), ATF4 (E), Chop (F), ATF6 (G), IRE1 (H), XBP1 (I), GCLM $(\mathrm{J})$ and $\mathrm{HO}-1(\mathrm{~K})$. Significant statistical decreases were indicated with $\#, p<0.01$, whereas significant increases were represented by ${ }^{*} p<0.01$ and ${ }^{* *} p<0.001$. 
Close examinations of other two ER signaling arms ATF6 and IRE1 showed distinct time-dependent induction of their mRNA expression by TU treatment of $\mathrm{Nrf1} / 2^{+/+}$cells, respectively with different peaks at $12 \mathrm{~h}$ or $20 \mathrm{~h}$ (Figure $3 \mathrm{G}$, 3H). Such significantly TU-induced increases in ATF6 mRNA expression were substantially suppressed in Nrf1 $\alpha^{-1-}$ cells, and also partially inhibited by $N r f 2^{-/-\Delta T A}$, but exhibited almost no changes in caNrf2 ${ }^{\Delta N}$ cells (Figure $\left.3 G\right)$. This indicates that Nrf1 $\alpha$ is required for regulating transcriptional expression of ATF6 gene induced by TU, while Nrf2 only makes a minor contribution to this response. By contrast with $N r f 1 / 2^{+/+}$cells, induction of IRE1 mRNA expression by TU was obviously prevented in $\mathrm{Nrf1} \alpha^{-/-}$cells (with hyper-expression of Nrf2), but not recovered in $\mathrm{Nrf2} 2^{-/-\Delta \mathrm{TA}}$ cells (Figure $3 \mathrm{H}$ ), implying a major contribution of $\mathrm{Nrf1} \alpha$, rather than $\mathrm{Nrf2}$, to IRE1 transcriptional expression. As such, the late-stage induction of IRE1 after $20 \mathrm{~h}$ TU treatment was also reduced by $N r f 2^{-1-\Delta T A}$. Contrarily, caNrf2 ${ }^{\Delta N}$ led to rather significant elevations in basal and TU-inducible IRE1 mRNA levels, with an early higher peak that stimulated at 8-h TU treatment and maintained to $12 \mathrm{~h}$, after being abruptly lowered to similar levels to the late-stage induction of $\mathrm{Nrf1} / 2^{+/+}$cells (Figure $3 \mathrm{H}$ ). These suggest that dual opposing roles of $\mathrm{Nrf2}$ in the IRE1 transcriptional response to TU may depend on the presence of distinct functional domains within this CNC-bZIP factor and/or their biochemical modifications by the putative signaling, albeit the detailed mechanisms are unknown. Besides, transcriptional expression of XBP1 mRNA as a direct substrate of IRE1 was further analyzed by qRT-PCR. The results disclosed that an accelerated increase in XBP1 mRNA expression to a 4-fold maximal value was induced by TU after 8-h treatment of $\mathrm{Nrf1/2} 2^{+/+}$cells (Figure 3I). By comparison with wild-type, TU-inducible XBP1 expression was significantly decreased in $\mathrm{Nrf1} \alpha^{-/-}$cells, and also partially reduced by $N r f 2^{-1-\Delta T A}$ or caNrf2 $2^{\Delta N}$. Hence, it is postulated that Nrf1 $\alpha$ and $\mathrm{Nrf2}$ are involved in co-regulating the responsive expression of the XBP1 gene to TU.

Next, examinations of ARE-driven genes by qRT-PCR revealed that TU treatment caused distinct time-dependent induction of GCLM and $\mathrm{HO}-1$ expression in $\mathrm{Nrf1} / 2^{+/+}$cells (Figure 3J,3K). By contrast, induction of GCLM and $\mathrm{HO}-1$ by TU was further significantly incremented in $\mathrm{Nrf1}^{-/-} \alpha^{-}$cells (retaining hyper-expression of Nrf2). Furtherly, caNrf2 ${ }^{\Delta N}$ also gave rise to substantial increases in basal and TU-induced mRNA levels of GCLM, rather than HO-1 (Figure 3J,3K). Conversely, Nrf2 $2^{-1-\Delta T A}$ led to obvious decreases in basal and TU-induced mRNA levels of HO-1, but not GCLM (Figure 3J,3K). These imply that transcriptional responses of $\mathrm{HO}-1$ and GCLM to TU are mediated dominantly by Nrf2, albeit Nrf1 partially contributes to this antioxidant response. In addition, TU-inducible expression of $\mathrm{HO}-1$ is also partially reduced by caNrf2 $2^{\Delta N}$ (Figure 3K), implicating a possible attribution to the loss of the Neh2 domain from Nrf2. Taken altogether, our results demonstrate that transcriptional expression of Nrf1 and Nrf2, as well as co-target genes GCLM and $\mathrm{HO}-1$, is differentially induced by ER stressor, as accompanied by distinct activation of UPR signaling networks.

Distinct contributions of Nrf1 $\alpha$ and Nrf2 to protein expression of antioxidant genes induced by ER stressor. Here, we further determined changes in the protein expression of Nrf1 and Nrf2, along with downstream antioxidant genes GCLM and HO-1, in different genotypic cellular responses to TU stress stimulation for distinct lengths of time from $4 \mathrm{~h}$ to $24 \mathrm{~h}$. Western blotting of $\mathrm{Nrf1} / 2^{+/+}$cells that had been treated TU (as a specific inhibitor of oligosaccharyl transferases to block N-glycosylation of newly-synthesized polypeptides) revealed that abundance of the full-length Nrf1 glycoprotein-A was gradually decreased from 8-h to its disappearance (Figure 4A1). Instead, the abundances of deglycosylated and processed Nrf1 protein-C/D were relatively incremented as the TU treatment time was increased. By sharp contrast, all these Nrf1-derived isoforms were completely abolished by specific knockout of $\mathrm{Nrf1} \alpha^{-/-}$(Figure 4B1). In addition, Nrf1 $\alpha$-derived protein levels were also modestly influenced by $N r f 2^{-1-\Delta T A}$ or caNrf2 ${ }^{\Delta N}$, because both 
mutants gave rise to an obviously-accelerated disappearance of Nrf1 glycoprotein within $4 \mathrm{~h}$ to $8 \mathrm{~h}$ of TU treatment (Figure 4C1,4D1), when compared with its presence in $\mathrm{Nrf1} / 2^{+/+}$cells (Figure 4A1). Conversely, this disappearance of Nrf1 glycoprotein was, rather, replaced by accelerated abundances of Nrf1 protein-C/D in caNrf2 ${ }^{\Delta N}$ cells (Figure 4D1). Together, these results demonstrate that glycosylation, deglycosylation and proteolytic processing of Nrf1 (associated with the ER) are regulated by TU-induced stress response signaling, part of which may be mediated by Nrf2.
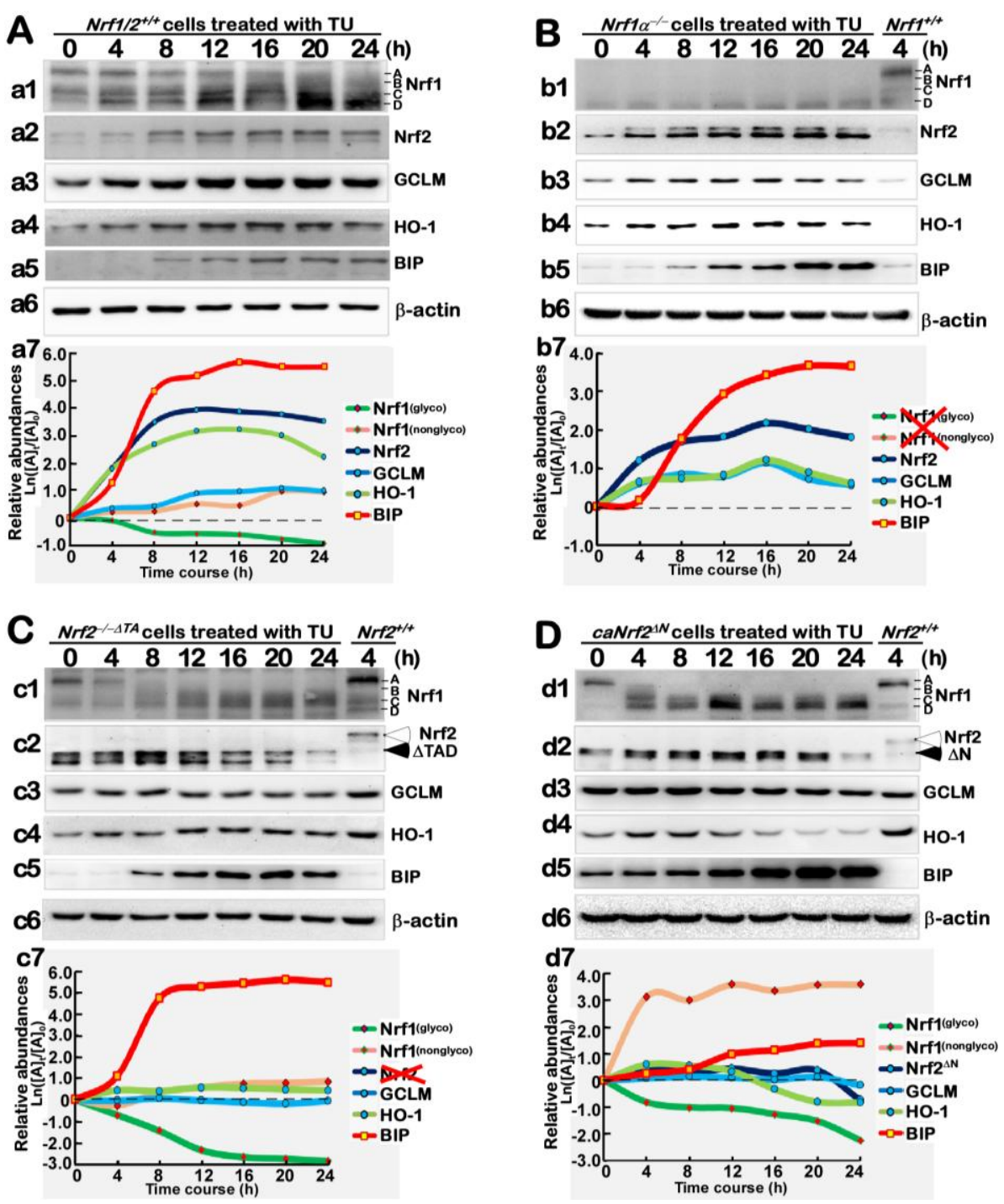

Figure 4. TU-inducible changes in the protein expression of antioxidant genes.

Distinct cell lines of $N r f 1 / 2^{+1+}(\mathrm{A}), N r f 1 \alpha^{-{ }_{-}}(\mathrm{B}), N r f 2^{-1-\Delta T A}(\mathrm{C})$ and $c a N r f 2^{\Delta N}$ (D) were treated with $2 \mu \mathrm{g} / \mathrm{ml} \mathrm{TU}$ for the indicated times from 0 to $24 \mathrm{~h}$. The TU-inducible changes in the protein expression of distinct responsive genes were determined by Western blotting with the indicated antibodies against Nrf1, Nrf2, GCLM, HO-1, BIP/GRP78 or $\beta$-actin. The intensity of blots representing different protein expression levels were also quantified by the Quantity One 4.5 .2 software and shown graphically.

Indeed, it is true that a gradual increment in Nrf2 protein abundances resulted from 4-h to 24-h TU-treatment of $\mathrm{Nrf1} / 2^{+/+}$cells (Figure 4A2). More intriguingly, a major processed isoform of Nrf2 was gradually incremented with the increasing time of TU treatment of $\mathrm{Nrf1}^{-\alpha^{--}}$cells (Figure 4B2). Similar observations was also represented in 
$\operatorname{caNrf2^{\Delta N}}$, but not $N r f 2^{-1-\Delta T A}$ cells (c.f. Figure 4D2 with 4C2). Hence, it is inferable that a putative proteolytic processing of Nrf2 may occur through within its N-terminal Neh2 domain, albeit the detailed mechanism requires to be elucidated.

Further examinations of antioxidant protein expression unraveled that distinct time-dependent increments of GCLM and HO-1 were significantly induced by TU treatment in $N r f 1 / 2^{+/ t}$ cells (Figure $\left.4 A 3,4 A 4\right)$. Similar induction of GCLM and HO-1 by TU was also observed in Nrf1 $\alpha^{-1-}$ cells (Figure 4B3,4B4), but was obviously reduced by $\mathrm{Nrf2}{ }^{-/-\Delta T A}$ (Figure 4C3, 4C4), with compared with the controls from $\mathrm{Nrf1} / 2^{+/+}$cells. However, caNrf2 ${ }^{\Delta N}$ cells displayed almost no changes in GCLM protein (Figure 4D3); this was accompanied by a modest decrease in HO-1 expression (Figure 4D4). Collectively, these results demonstrate that, although Nrf2 is negatively regulated by Nrf1, the former Nrf2 makes a major contribution to regulating expression of GCLM and $\mathrm{HO}-1$ genes, possibly through its N-terminal Neh2 domain.

Next, Western blotting of the ER stress-responsive chaperone BIP showed its protein abundance was increased in a time-dependent manner from $4 \mathrm{~h}$ to $24 \mathrm{~h}$ of TU treatment of $\mathrm{Nrf1} / 2^{+/+}$cells (Figure 4A5). Interestingly, remarkable increments in BIP protein levels were presented in TU-treated $N r f 1 \alpha^{-/-}$and $N r f 2^{-1-\Delta T A}$ cells (Figure 4B5,4C5), albeit its mRNA expression levels were lowered to different extents in these two cell lines (Figure 3C). In addition, basal and TU-stimulated BIP abundances were also strikingly incremented in caNrf2 ${ }^{\Delta N}$ cells (Figure 4D5). Overall, these indicate that Nrf1 $\alpha$ and Nrf2 are not essential for mediating BIP protein expression, albeit both CNC-bZIP factors are involved in this chaperone transcriptional response to TU, besides antioxidant response to this ER stressor.

Distinct involvements of Nrf1 $\alpha$ and Nrf2 in differential expression of TU-induced ER stress responsive genes.

Clearly, it is known that the chaperone BIP protein is a key sensor to ER stress induced by TU, so that expression of its cognate gene as a direct effector is activated in UPR [1-4]. Such an ER stress model was also successfully constructed as described above. Herein, we further examined whether Nrf1 $\alpha$ and Nrf2 are required for crucial protein expression for the UPR signaling cascades. As anticipated, the phosphorylated protein levels of PERK were significantly increased following $12 \mathrm{~h}$ to $16 \mathrm{~h}$ of TU treatment of $\mathrm{Nrf1} / 2^{+/+}$cells (Figure 5A2). The induction of $\mathrm{p}$-PERK by TU appeared to be prevented in $N r f 1 \alpha^{-/-}$and $N r f 2^{-/-\Delta T A}$ cells (Figure 5B2, 5C2). By contrast, caNrf2 ${ }^{\Delta N}$ led to an early modest induction of $\mathrm{p}$-PERK by TU at $4 \mathrm{~h}$ to $8 \mathrm{~h}$ following treatment (Figure 5D2). These suggest that Nrf1 $\alpha$ and Nrf2 may be required for regulation of the PERK signaling response to TU.

Meanwhile, the total protein abundances of elF2 $\alpha$ (as a main substrate of p-PERK to yield p-elF2 $\alpha$ ) were almost unaltered by $\mathrm{TU}$ in all the above-described four cell lines (Figure 5A4, 5B4, 5C4, 5D4). In addition to the basal elF2 $\alpha$ auto-phosphorylation, its TU-inducible phosphorylation was also enhanced as the time of treatment was increased from $4 \mathrm{~h}$ to $16 \mathrm{~h}$, and then maintained until $24 \mathrm{~h}$ after treatment of $\mathrm{Nrf1} / 2^{+/+}$cells (Figure 5A3). However, TU-induced elF2 $\alpha$ phosphorylation was almost unaffected in $\mathrm{Nrf1}^{-/-}$cells (Figure 5B3), when compared with the control of 4-h TU-treated $\mathrm{Nrf1} / 2^{+/+}$cells. Conversely, induction of elF2 $\alpha$ phosphorylation by TU was partially recovered in $\mathrm{Nrf} 2^{-/-\Delta T A}$ cells (Figure 5C3). These results indicate that Nrf1 $\alpha$ and Nrf2 may contribute to positive and negative regulation of elF2 $\alpha$ induction by TU, respectively. Yet, caNrf2 ${ }^{\Delta N}$ gave rise to an increase in basal elF2 $\alpha$ auto-phosphorylation and its TU-inducible phosphorylation by $20 \mathrm{~h}$ of treatment (Figure 5D3), by comparison with the control of 4-h TU-treated $\mathrm{Nrf1} / 2^{+/+}$cells. This intriguing data implicates that elF2 $\alpha$ may be negatively regulated by the $\mathrm{N}$-terminal Neh2 domain of Nrf2, besides its transactivation domains (because both lacked in caNrf2 ${ }^{\Delta N}$ and $N r f 2^{-1-\Delta T A}$ cells, respectively). 

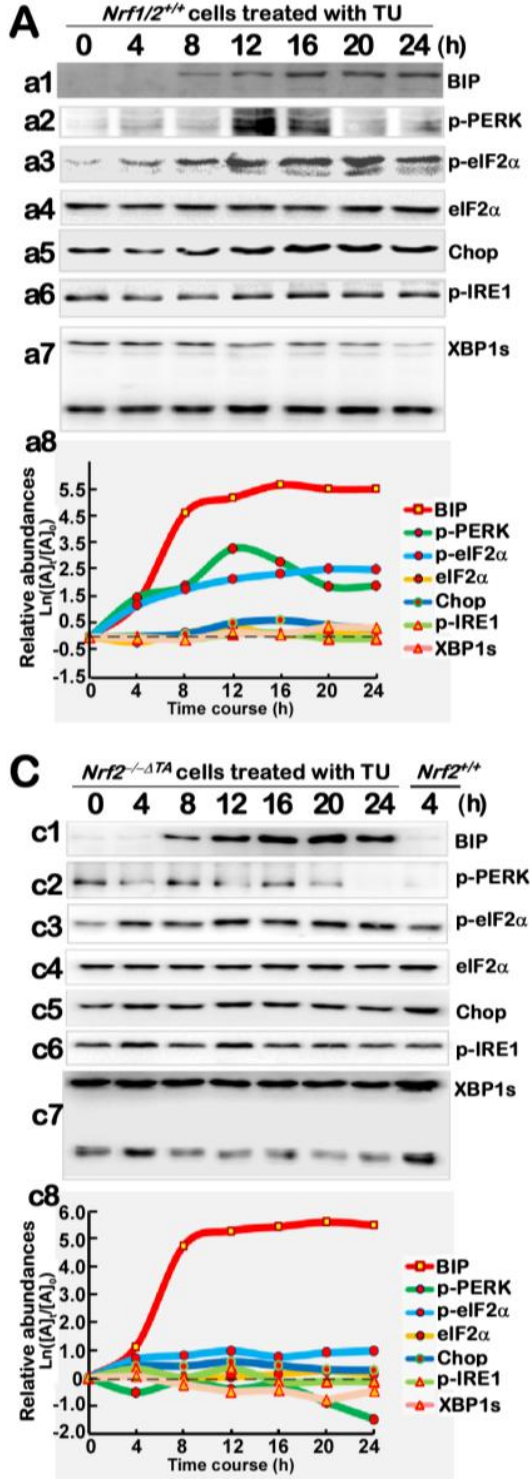
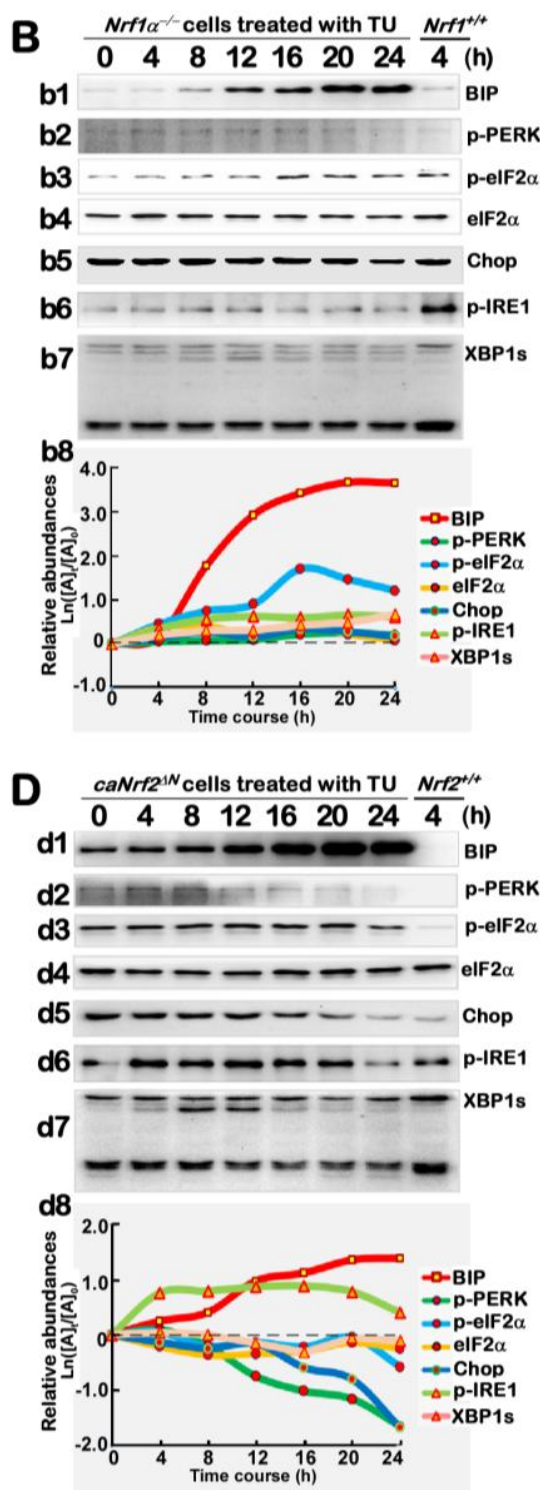

Figure 5. TU-inducible changes in the protein expression of ER stress-responsive genes.

Distinct cell lines of $N r f 1 / 2^{++}(A), N r f 1 \alpha^{-1-}(B), N r f 2^{-1-\Delta T A}$ (C) and caNrf2 ${ }^{\Delta N}$ (D) were treated with $2 \mu \mathrm{g} / \mathrm{ml}$ of TU for the indicated times from 0 to $24 \mathrm{~h}$. The TU-inducible changes in the protein expression of distinct responsive genes were determined by Western blotting with the indicated antibodies against BIP/GRP78 (as a positive reference, that was duplicated from Figure 4), p-PERK, p-eIF2 $\alpha$, elF2 $\alpha$, Chop, p-IRE1 or XBP1. The intensity of blots representing different protein expression levels were also quantified by the Quantity One 4.5.2 software and shown graphically.

Western blotting examination of Chop, as an effector of the PERK-elF2 $\alpha$-ATF4 signaling pathway, revealed that a modest increment in Chop protein levels resulted from $8 \mathrm{~h}$ to $24 \mathrm{~h}$ of TU treatment of $\mathrm{Nrf1} / 2^{+/+}$cells (Figure 5A5). By

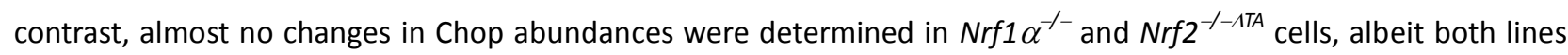
had been treated with TU (Figure 5B5,5C5). However, basal Chop abundance was obviously elevated by caNrf2 $2^{\Delta N}$ and appeared to be almost unaffected by TU within $12 \mathrm{~h}$ after this treatment, but thereafter decreased gradually to a similar level to the control of 4-h TU-treated $\mathrm{Nrf1}_{1} / 2^{+/+}$cells (Figure 5D5). Together, these results indicate that Chop is co-regulated by Nrf1 $\alpha$ and Nrf2, but the latter Nrf2 may also contribute to negative regulation of Chop possibly by its Neh2 domain, in the ER-to-nuclear response to the long-term TU-induced stress. 
Next, distinct time-dependent expression of the IRE1/ATF6-XBP1 signaling molecules was examined by Western blotting. The results unraveled that, since the existing auto-phosphorylation of IRE1 has been at a considerable high level, TU only induced a modest increase in its phosphorylated abundance following 12-h treatment of $\mathrm{Nrf1/2}{ }^{+/+}$cells (Figure 5A6). Such low induction of IRE1 by TU appeared to be almost abolished in $\mathrm{Nrf1} \alpha^{-/-}$cells (Figure 5B6, when compared to the control of 4-h TU-treated $\mathrm{Nrf1} / 2^{+/+}$cells), but also was only slightly recovered from $4 \mathrm{~h}$ to $12 \mathrm{~h}$ of TU treatment of $\mathrm{Nrf2}^{-/-\Delta T A}$ cells (Figure 5C6). However, caNrf2 ${ }^{\Delta N}$ cells raised a rather higher induction of phosphorylated IRE1 by TU (Figure 5D6). Together, these indicate that Nrf1 $\alpha$ and Nrf2 are involved in regulating the IRE1 signaling response to TU. In addition, it is important to note that the intact XBP1u mRNA, though as a direct substrate of IRE1 to yield a specifically-spliced XBP1s (besides its mRNA decay), is also transcriptionally regulated by ATF6 signaling in ER stress response [[1-4], Figure 1]. Therefore, Western blotting showed that a few of processed XBP1s bands were observed in $\mathrm{Nrf1} / 2^{+/+}$cells (Figure 5A7), and also obviously enhanced by TU in $\mathrm{Nrf1} \alpha^{-1-}$ or caNrf2 ${ }^{\Delta N}$ cells (Figure 5B7, 5D7), but not determined in $N r f 2^{-1-\Delta T A}$ cells (Figure 5C). Conversely, the putative XBPu protein was rather reduced by $N r f 2^{-1-\Delta T A}$ only. These results implicate that Nrf2 may contribute to regulating XBP response to TU, albeit the detailed mechanism needs to be further elucidated.
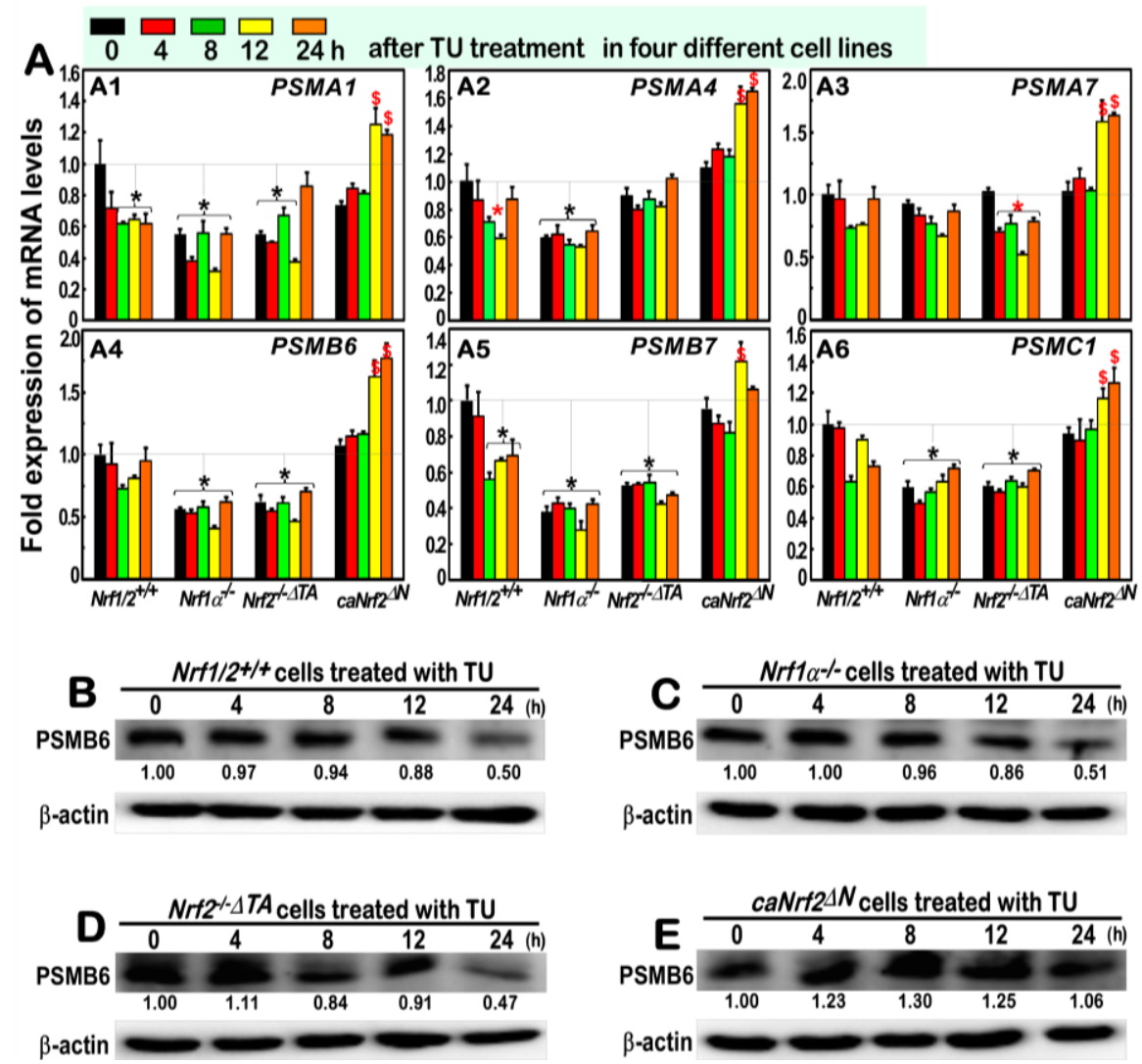

Figure 6. No obvious induction of some examined proteasomal genes by TU.

Distinct cell lines of $\mathrm{Nrf1} / 2^{+/+}, \mathrm{Nrf1} \alpha^{-1-}, \mathrm{Nrf2} 2^{-1-\Delta T A}$ and $\mathrm{caNrf2} 2^{\Delta N}$ were treated with $2 \mu \mathrm{g} / \mathrm{ml}$ TU for the indicated times from 0 to $24 \mathrm{~h}$. The TU-inducible mRNA levels of some proteasomal genes, including PSMA1, PSMA4, PSMA7, PSMB6, $P S M B 7$ and PSMC1, were determined by real-time $\mathrm{QPCR}(\mathrm{A})$, and Western blotting with indicated antibodies against PSMB6 or $\beta$-actin in different cell lines $N r f 1 / 2^{+/+}(B), N r f 1 \alpha^{-1-}(C), N r f 2^{-1-\Delta T A}$ (D) and caNrf2 $2^{\Delta N}$ (E). The intensity of blots representing PSMB6 was also quantified by the Quantity One 4.5 .2 software, and shown on the bottom. 
No induction of proteasomal (PSM) subunit genes regulated by Nrf1 in the response to TU stressor.

As described by us and others [22, 24, 29], it is rather clear that Nrf1, but not Nrf2, exerts an important biological role in the transcriptional expression of all PSM genes. Such transcriptional regulation of PSM genes by Nrf1 is also accompanied by induction of all three classic ER-driven stress response signaling pathways mediated by PERK1, IRE1 and ATF6, along with the proteasomal compensatory response to limited extents of proteasome inhibitors [24]. Here, we further examined whether TU-stimulated Nrf1 and Nrf2 are required for the ER-to-nuclear signaling responses to transactivate PSM genes. The results revealed that none of all six PSM mRNA expression levels was induced by TU in Nrf1/2 $2^{+/+}$cells (Figure 6A). Contrarily, PSMA1 and PSMB7 mRNA levels were obviously down-expressed in TU-treated $\mathrm{Nrf1} / 2^{+/+}$cells (Figure 6A1, 6A5).

Further qRT-PCR analysis of Nrf1 $\alpha^{-1-}$ cells unraveled that the basal mRNA expression levels of PSMA1, PSMA4, PSMB6, PSMB7 and PSMC1, but not PSMA7, were significantly down-regulated and also unaffected by TU stimulation (Figure 6A). Similar down-regulation of PSMA1, PSMB6, PSMB7, PSMC1, but not PSMA4 or PSMA7, was detected in $N r f 2^{-1-\Delta T A}$ cells. However, all these six PSM genes were only induced by the long-term exposures of TU in $c a N r f 2^{\Delta N}$ cells from $12 \mathrm{~h}$ to $24 \mathrm{~h}$. Moreover, Western blotting of PSMB6 disclosed that its protein abundance was decreased by TU, with the increasing treatment time of $\mathrm{Nrf1} / 2^{+/+}$cells (Figure 6B). Similarly TU-triggered decreases in PSMB6 were almost unaltered by $\mathrm{Nrf1} \alpha^{-/-}$or $N r f 2^{-/-\Delta T A}$ cells (Figure 6C,6D), but markedly reversed by caNrf2 ${ }^{\Delta N}$ cells with a modest increase (Figure 6E). Collectively, distinct contributions of Nrf1 $\alpha$ and Nrf2 to basal, but not TU-inducible, expression of some PSM genes are demonstrated. Yet, Nrf2 might exert opposing roles in this event, depending on its functional domains within different responsive contexts.

\section{Discussion}

In the present study, it is demonstrated that there exists a bi-directional cross-talk between UPR-triggered signaling and ARE-driven cytoprotective responses to the ER stressor TU (Figure 7). Importantly, we have also demonstrated the evidence that opposite roles of Nrf1 and Nrf2 are unified to coordinate different cellular responses to TU, leading to differential activation of ER-driven stress signaling networks. Of note, loss of Nrf1 down-regulates expression of antioxidant, detoxification and particularly proteasomal genes, leading to severe oxidative stress and concurrently ER stress [27, 34]. The latter pathophysiological event is primarily attributable to disruption of protein folding within the ER and dysfunction of ERAD, such that unfolded and misfolded proteins, along with oxidized and damaged proteins, are accumulated within the ER. Consequently, the canonical UPR signaling pathways are activated by PERK, IRE1 and ATF6 (Figures 1 and 7), in order for the ER adaptive remodeling to diminish loading of nascent polypeptides, remove aberrant folded proteins, and then restore itself biological function of this organelle [35, 36]. This notion is supported by the evidence showing that endogenous ER stress signaling to activate UPR occurred in the steatotic hepatocytes with a homozygous knockout of $\mathrm{Nrf1}^{-1-}$ [27], but not $\mathrm{Nrf2}^{-t_{-}}$[12] and similar ER stress-inducible response was further enhanced by proteasomal inhibition of the heterozygous $\mathrm{Nrf1}^{+/-}$livers, when compared with wild-type [27]. Thereby, Nrf1 plays an essential role in maintaining the ER homeostasis, but its functional loss results in the ER transformation and proliferation of $\mathrm{Nrf1}^{-1-}$ cells in conditional knockout mice, that are spontaneously developed with non-alcoholic steatohepatitis (NASH) and liver cancer [34]. Such phenotypes should be embodied as a pathological consequence of chronic stimulation of ER stress and prolonged activation of UPR signaling, as occurred concomitantly with severe oxidative stress, which together lead to carcinogenesis ultimately [37-39]. 


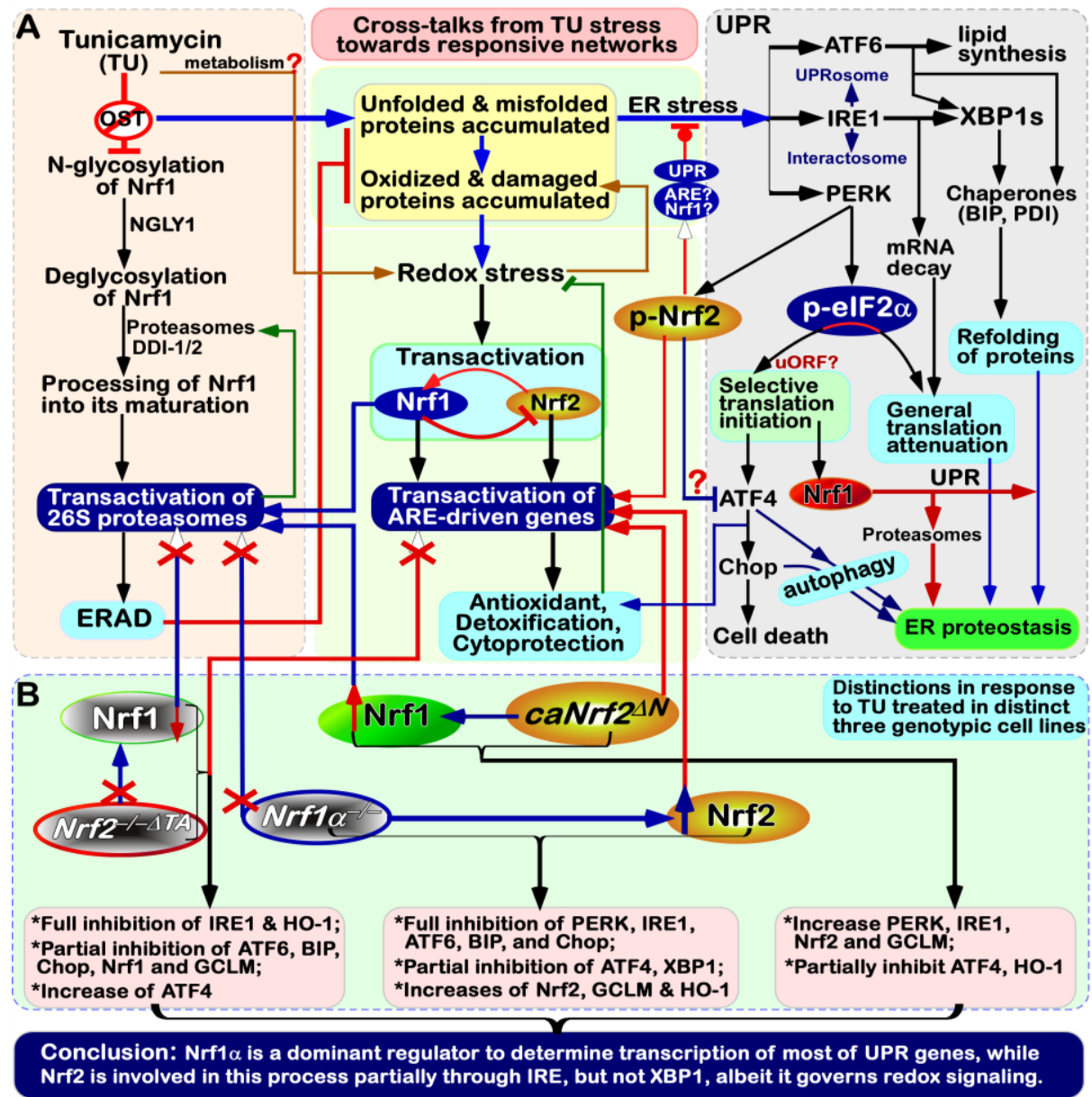

Figure 7. Cross-talks from TU stress signaling to different ER-to-nuclear responsive networks.

(A) There exist multiple cross-talks from TU stress signaling to different ER-to-nuclear responsive networks. Inhibition of Nrf1 N-glycosylation by TU leads to subsequent blockage of its deglycosylation and proteolytic processing to yield a mature CNC-bZIP factor, before transactivating proteasomal genes. Such disruption of Nrf1-mediated proteasomal degradation (e.g., ERAD) leads to aberrant accumulation of oxidized, damaged and misfolded proteins, in addition to TU-led non-glycosylated and unfolded proteins. These together result in classic ER stress along with redox stress. Of note, distinct and even opposing roles of Nrf1 and Nrf2, as well as both inter-regulatory effects on cytoprotection against ER redox stress, are integrally unified in different cellular signaling responses within distinct gene-regulatory networks. (B) Our evidence demonstrates that Nrf1 acts as a dominant regulator of most of UPR-target genes, apart from its negative regulation of Nrf2. By contrast, Nrf2, besides as a direct substrate of PERK, is also involved in this response to TU partially through the IRE1 signaling pathway, but not by its downstream XBP1. Although Nrf2 governs transcription of Nrf1 and their co-target antioxidant genes, it also contributes to negative regulation of ATF4, that is selectively translated by the elF2 $\alpha$-based machinery. As described for ATF4, Nrf1 is predicted to contain an upstream open reading frame (uORF) within its full-length mRNA transcript.

Notably, Nrf1 is a moving transmembrane protein with dynamic membrane-topologies (that are distinctive from those of PERK, IRE1 and ATF6 within and around the ER, as deciphered in Figure 1), and its topovectorial processes determine its post-synthetic modification and its trans-activity to mediate target PSM genes [24, 32]. Interestingly, our evidence has also been provided showing that the canonical UPR signaling by PERK, IRE1 and ATF6 to differential expression of distinct responsive genes is activated by classic ER stressor TU; this is accompanied by transcriptional expression of $\mathrm{Nrf1}$ and $\mathrm{Nrf2}$, as well as their co-target GCLM and $\mathrm{HO}-1$ (Figure 7). This appears to raise a paradoxical 
question about Nrf1, because N-glycosylation of this CNC-bZIP protein (that is newly synthesized in the ER lumen) is sufficiently blocked by TU, with secondary inhibition of its ensuing deglycosylation and proteolytic processing to yield an N-terminally-truncated mature factor in close proximity to membranes. This inhibitory effect of TU on Nrf1 is also further supported by no induction of all examined PSM genes regulated by Nrf1, rather than Nrf2, leading to ERAD dysfunction so as to exacerbate accumulation of those misfolded, oxidized and damaged proteins by this stressor TU. In a feedback regulatory response to mitigate this deteriorating ER stress, Nrf1 is transcriptionally activated by Nrf2. This is based on the fact that Nrf1 is identified as a direct target gene of Nrf2 [31] and also Nrf2, as a direct substrate of PERK, is activated by TU [10-12]. Further translational expression of Nrf1 could also be selectively initiated by a putative mechanism accounting for ATF4, c-Myc and C/EBP [40-42] (Figure 7), which can enable their translational expression to switch from their upstream open reading frames (uORF) to their main open reading frames (mORF), in the cellular response to ER stress [8]. Such being the case, it is hence postulated that such uORF (and/or its products) could act as a molecular pathophysiological switch driving carcinogenesis or other degenerative diseases.

Although the above relevant detailed mechanisms warrant to be further elucidated, our evidence has been here presented demonstrating that Nrf1 is significantly increased at both its transcriptional and translational expression levels in TU-stimulated of $\mathrm{Nrf1} / 2^{+/+}$cells, particularly of caNrf2 $2^{\Delta N}$ cells (with an enhanced constitutive expression of Nrf2). This implies that a portion of non-glycosylated Nrf1 and its subsequent processed isoforms are incremented in a feedback regulatory circuit. However, it is inferable that a fraction of such non-glycosylated and processed proteins of Nrf1 (that are generated during the TU-stress conditions) only has a weak activity to mediate target genes, relative to another distinctive fraction of its deglycosylated and processed proteins (that are generated in TU-untreated cells). In fact, this point is convincingly evidenced by inhibition of peptide:N-glycosidase (PNG-1/NGLY1, an evolutionarily conserved deglycosylation enzyme) to inactivate Nrf1 and thus down-regulate expression of its target PSM genes, so as to potentiate cytotoxicity of proteasomal Inhibitors [43]. Besides, the NGLY1-NRF1 pathway is revealed to exert an additional novel functions in mitochondrial homeostasis and inflammation pathogenesis [44]. Similar inactivation of Nrf1 to disrupt deglycosylation of misfolded N-glycosylated proteins has been recently unveiled by loss-of-function of NGLY1, a homozygous mutant condition that has been characterized by a complex neurological syndrome, hypo- or alacrimia, and elevated liver transaminases $[45,46]$. Similarly, the functional analysis of a Drosophila model of NGLY1 deficiency to its CNC inactivation provides insight into therapeutic approaches [47]. Moreover, additional homologue SKN-1A associated with the ER of Caenorhabditis elegans also mediates a cytoplasmic unfolded protein response and promotes longevity [48]. This biological event occurs to be regulated by the amino acid sequence editing of SKN-1A by NGLY1 from its glycosylated Asn (-N-S/T-) into acidic Asp (-D-S/T-) residues within this protein, in order to control expression of PSM genes against proteotoxic stress [49]. Overall, these supportive findings are prime to confirm our prior work on Nrf1 glycosylation and deglycosylation to finely tune its transactivation activity [20, 33, 50, 51].

Moreover, it should also be noted that the dynamic membrane-topology of Nrf1 to determine its post-synthetic processing and its transactivation activity might be modulated by cholesterol within membranes in the ER-to-nuclear signaling response to cellular stress. This viewpoint is attributable to direct binding of Nrf1 to cholesterol by its five cholesterol-recognized amino acid consensus (CRAC) sites within this CNC-bZIP protein [32, 51, 52]. Recently, the ER-associated Nrf1 is also identified as a vital sensor to cholesterol within membranes [53]. These collective findings demonstrate that Nrf1 plays a central role in maintaining cholesterol homeostasis by a negative feedback regulatory 
loop against an additional ER membrane-bound SREBP2 (sterol response element binding protein). In reality, the UPR signaling mediated by SREBP (and ATF6) has also been showed a linkage to a lipid stress response [54-56]. Activation of SREBP signaling occurs only after being translocated into the Golgi apparatus, in which it is subject to sequential cleavages by Site-1 and Site-2 proteases in a similar fashion to the case of ATF6 (Figure 1). However, Nrf1 is neither translocated into the Golgi apparatus and nor cleaved by both Site-1 and Site-2 proteases [33, 50]. As a master of fact, the bona fide activation of Nrf1 occurs only after its dynamic flipping out of ER membranes and then by its topologyregulated juxtamembrane proteolytic processing by cytosolic proteasomes and DDI-1/2 proteases to yield a mature CNC-bZIP factor $[8,24,32,57]$. Besides, SREBP1 was also identified as a direct upstream regulator of Nrf1, which is involved in the mTORC1 signaling response to insulin and epidermal growth factor [23]. Yet, it remains unknown whether there exists a coordinated cross-talk between oxidative stress response and lipid-coupled UPR. As such, it is plausible that Nrf1 senses cholesterol changes in the vicinity of ER membranes, and relevant signals are integrated and transduced to down-regulate cognate target genes. Meanwhile, the cytoprotective responsive signaling to ER stress enables Nrf1 to be selectively translated by the putative uORF existing within the first exon of its full-length transcripts and/or processed by its topology-regulated juxtamembrane proteolysis. Such a unique mechanism is definitely distinctive from that accounts for both ATF6 and SREBP1/2 factors in the ER stress responses to unfolded proteins and overloaded lipids.

Here, it is of a crucial significance to reveal that specific knockout of $N r f 1 \alpha^{-1-}$ leads to aberrant hyper-expression of Nrf2 and cognate target GCLM and HO-1, as companied by down-expression of most UPR-target genes (referenced

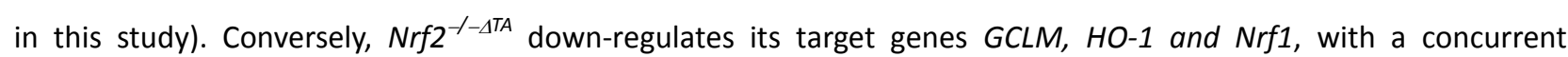
inhibition of some UPR-target genes, such as IRE1, ATF6, BIP and Chop. However, ATF4 is strikingly up-regulated by inactivation of Nrf2, while their upstream PERK expression is almost unaffected. These findings, together with our previous work [24,31], demonstrate that Nrf1 $\alpha$ does not only dictate specific functioning of Nrf2 to be exerted in antioxidant responses, and also act as a dominant player in the regulation of most UPR-target genes (that contain the ARE sequences within their promoter regions, as listed in Table S1). Also, Nrf2 is partially involved in mediating the expression of some UPR genes by controlling transcription of Nrf1 and IRE1, but not XBP1. However, expression of ATF4 is down-regulated by caNrf2 ${ }^{\triangle N}$, albeit with up-regulation of PERK (and IRE1). This implies a putative mechanism for Nrf2 (as a direct substrate of PERK) to compete against the PERK-eIF2 $\alpha$-ATF4 signaling (Figure 7). Furthermore, a partial inhibition of $\mathrm{HO}-1$ by caNrf2 $2^{\Delta N}$ also indicates a constitutive loss of its N-terminal Neh2 function as an extra unidentified nuclear transactivation domain after Nrf2 enters the nucleus.

\section{Conclusion}

The evidence has been herein presented demonstrating a unity of distinct and even opposing roles of Nrf1 and Nrf2 in discrete cellular responses to ER stress induced by TU, hence leading to differential activation of the ER-to-nuclear signaling networks. Notably, there exist multiple cross-talks between UPR- and ARE-driven signaling cascades, aiming to maintain cellular redox, protein and lipid homeostasis. Altogether, these responses can make cell fate decisions to be modulated under ER stress conditions [58], as discovered for its homologous Skn-1 in Caenorhabditis elegans [18]. Importantly, Nrf1 $\alpha$ (and/or its derivates) is a dominant regulator to determine the transcriptional expression of most of UPR-target genes, whereas Nrf2 is also involved in this ER-to nuclear response partially through IRE1 but not its substrate XBP1, albeit it is a central player in governing redox signaling networks. 


\section{Materials and Methods}

Cell lines and Reagents.

The human hepatocellular carcinoma HepG2 cells (i.e. $N r f 1 / 2^{t /+}$ ) were obtained originally from the American Type Culture Collection (ATCC, Manassas, VA, USA). Three derived cell lines with knockout of $N r f 1 \alpha^{-/-}$or $N r f 2^{-/-\Delta T A}$ and a constitutive activation of $\mathrm{Nrf2}$ (i.e. caNrf2 $2^{\Delta N}$ ) were established by Qiu et al [31]. They were cultured in a $37^{\circ} \mathrm{C}$ incubator with 5\% carbon dioxide, and allowed for growth in Dulbecco's modified Eagle's medium (DMEM) with 25 $\mathrm{mmol} / \mathrm{L}$ high glucose, $10 \%$ (v/v) fetal bovine serum (FBS), 100 units/ml penicillin-streptomycin. TU (with MW 816.89) was purchased from Sangon Biotech Co., Ltd. (Shanghai, China). All pairs of primers used for qRT-PCR analysis are listed in Table S2.

Cell viability.

All four cell lines $\mathrm{Nrf1} / 2^{+/+}, \mathrm{Nrf1} \alpha^{-/-}, \mathrm{Nrf2} 2^{-1-\Delta T A}$ and caNrf2 ${ }^{\Delta N}$ were cultured for $24 \mathrm{~h}$ in DMEM containing $25 \mathrm{mmol} / \mathrm{L}$ glucose and $10 \%$ FBS. After reaching $70 \%$ of their confluences, they were then allowed for growth in fresh media with different concentrations of TU (at $0,0.5,1,2,4$, or $8 \mu \mathrm{g} / \mathrm{ml}$ ). For their time-course, they were also treated with $2 \mu \mathrm{g} / \mathrm{ml}$ of TU for different lengths of time (i.e. $0,4,8,12,16,20$, or $24 \mathrm{~h}$ ). The cell viability was evaluated by using an MTT-based cell proliferation and cytotoxicity assay kit (Beyotime, China).

The constitutive expression of ER stress-related genes in selected cell lines.

Equal amounts of $\mathrm{Nrf1} / 2^{+/+}, \mathrm{Nrf1} \alpha^{-/-}, \mathrm{Nrf2}{ }^{-1-\Delta T A}$, and $\mathrm{caNrf2} 2^{\Delta N}$ were cultured in 6-well plates before being harvested in a lysis buffer [59]. Total cell lysates were subjected to protein separation by SDS-PAGE gels containing $8 \%$ polyacrylamide, followed by Western blotting with antibodies against Nrf1 (made in our laboratory) and Nrf2 (from ABCAM, Cambridge, UK) or $\beta$-Actin (from Zhong Shan Jin Qiao Co., Beijing, China). The $\beta$-Actin served as an internal control to verify amounts of proteins that were loaded in each of wells. Meantime, a portion of differential expression genes were identified by transcriptome sequencing, and their relative basal expression levels were also calculated and presented as fold changes (mean \pm SD) in the RPKM (Reads Per Kilobase per Million mapped reads). According to the Log2-based RPKM values against those determined from $\mathrm{Nrf1} / 2^{+/+}$, the heatmaps for experimented cell lines were generated by using the MEV4.9 program.

The mRNA expression of examined responsive genes to TU.

After reaching $70 \%$ confluence of $N r f 1 / 2^{+1+}, N r f 1 \alpha^{-1-}, N r f 2^{-1-\Delta T A}$ and $c a N r f 2^{\Delta N}$ cell lines grown in DMEM containing 25 $\mathrm{mmol} / \mathrm{L}$ glucose and $10 \% \mathrm{FBS}$, they were treated for different time periods with $2 \mu \mathrm{g} / \mathrm{ml}$ of TU. Their total RNAs were extracted by using an RNA extraction kit (TIANGEN, China) and then subjected to reactions with reverse transcriptase (Promega, USA) to synthesize the single-strand cDNAs. Subsequently, relative mRNA expression levels of both ER stress-related and proteasomal genes in these cell lines were measured by RT-qPCR. This reaction was carried out in the GoTaq ${ }^{\circledR}$ real-time PCR detection systems, loaded on a CFX96 instrument (Bio-rad, USA). The results were analyzed by the Bio-Rad CFX Manager 3.0 software.

The protein expression of examined responsive genes to TU.

After reaching $70 \%$ confluence of $N r f 1 / 2^{+/+}, N r f 1 \alpha^{-\gamma_{-}}, N r f 2^{-\iota_{-\Delta T A}}$ and caNrf2 $2^{\Delta N}$ cell lines growth in DMEM containing $25 \mathrm{mmol} / \mathrm{L}$ glucose and 10\% FBS, they were treated with $2 \mu \mathrm{g} / \mathrm{ml}$ of TU from distinct lengths of time. Their total 
bioRxiv preprint doi: https://doi.org/10.1101/655159; this version posted May 31, 2019. The copyright holder for this preprint (which was not certified by peer review) is the author/funder. All rights reserved. No reuse allowed without permission.

lysates were separated by SDS-PAGE gels containing $10 \%$ polyacrylamide, followed by Western blotting with distinct primary antibodies against GCLM, HO-1, BIP, elF2 $\alpha$, Chop, p-IRE1, XBP1 and PSMB6. These antibodies were purchased from Abcam, Inc (Cambridge, UK), except that p-PERK and p-elF2 were from Cell Signaling Technology, Inc. (Massachusetts, USA). The $\beta$-Actin served as an internal control to verify amounts of proteins that were loaded in each of wells. The intensity of some immunoblots were quantified by the Quantity One 4.5 .2 software and shown graphically.

\section{Statistical analysis.}

The 'wet' experimental data provided in this study were represented as the mean \pm SD and were analyzed using the Student's $t$-test or Fisher's exact test, as appropriate. The resulting value of $p<0.05$ was considered as a significant difference. In addition, statistical determination of the 'dry' sequencing analysis was described by Wang, et al [60].

Data Availability: All data needed to evaluate the conclusions in the paper are present in this publication and/or the Supplementary Materials that can be found at xx. Additional data related to this paper may be requested from the authors.

Author contributions: Y.-P.Z., S.H. and X.R. performed most of both experiments and bioinformatics, and collected relative data. Z.Z. and Z.F. also did some experiments. L.Q. made experimental cell lines used in this study. Y.-P.Z. prepared draft of this manuscript with most figures and supplementary tables. Y.Z. designed this study, analyzed all the data, helped to prepare all figures with two cartoons, wrote and revised the paper.

Acknowledgments: The study was supported by the National Natural Science Foundation of China (NSFC, with key programs 91129703, 91429305 and project 81872336) awarded to Yiguo Zhang (Chongqing University, China).

Conflicts of Interest: The authors declare no conflict of interest. Besides, it should also be noted that the preprinted version of this paper had been initially posted at the bioRxiv xx; doi: xx on the xx of June, 2019.

\section{References}

1. Hegde, R. S.; Ploegh, H. L., Quality and quantity control at the endoplasmic reticulum. Curr Opin Cell Biol 2010, 22, (4), 437-46.

2. Wang, M.; Kaufman, R. J., Protein misfolding in the endoplasmic reticulum as a conduit to human disease. Nature 2016, 529, (7586), 326-35.

3. Grootjans, J.; Kaser, A.; Kaufman, R. J.; Blumberg, R. S., The unfolded protein response in immunity and inflammation. Nat Rev Immunol 2016, 16, (8), 469-84.

4. Preissler, S.; Ron, D., Early events in the endoplasmic reticulum unfolded protein response. Cold Spring Harb Perspect Biol 2019, 11, (4).

5. Pincus, D.; Chevalier, M. W.; Aragon, T.; van Anken, E.; Vidal, S. E.; El-Samad, H.; Walter, P., BiP binding to the ER-stress sensor Ire1 tunes the homeostatic behavior of the unfolded protein response. PLoS Biol 2010, 8, (7), e1000415.

6. Gogala, M.; Becker, T.; Beatrix, B.; Armache, J. P.; Barrio-Garcia, C.; Berninghausen, O.; Beckmann, R., Structures of the Sec61 complex engaged in nascent peptide translocation or membrane insertion. Nature 2014, 506, (7486), 107-10.

7. Eletto, D.; Chevet, E.; Argon, Y.; Appenzeller-Herzog, C., Redox controls UPR to control redox. J Cell Sci 2014, 127, (Pt 17), 3649-58.

8. Zhang, Y.; Xiang, Y., Molecular and cellular basis for the unique functioning of Nrf1, an indispensable transcription factor for maintaining cell homoeostasis and organ integrity. Biochem J 2016, 473, (8), 961-1000.

9. Ashabi, G.; Alamdary, S. Z.; Ramin, M.; Khodagholi, F., Reduction of hippocampal apoptosis by intracerebroventricular administration of extracellular signal-regulated protein kinase and/or p38 inhibitors in amyloid beta rat model of Alzheimer's disease: involvement of nuclear-related factor-2 and nuclear factor-kappaB. Basic Clin Pharmacol Toxicol 2013, 112, (3), $145-55$

10. Cullinan, S. B.; Zhang, D.; Hannink, M.; Arvisais, E.; Kaufman, R. J.; Diehl, J. A., Nrf2 is a direct PERK substrate and effector of PERK-dependent cell survival. Mol Cell Biol 2003, 23, (20), 7198-209.

11. Cullinan, S. B.; Diehl, J. A., Coordination of ER and oxidative stress signaling: the PERK/Nrf2 signaling pathway. Int $J$ Biochem Cell Biol 2006, 38, (3), 317-32.

12. Cullinan, S. B.; Diehl, J. A., PERK-dependent activation of Nrf2 contributes to redox homeostasis and cell survival following 
bioRxiv preprint doi: https://doi.org/10.1101/655159; this version posted May 31, 2019. The copyright holder for this preprint (which was not certified by peer review) is the author/funder. All rights reserved. No reuse allowed without permission.

endoplasmic reticulum stress. J Biol Chem 2004, 279, (19), 20108-17.

13. Bertolotti, A.; Zhang, Y.; Hendershot, L. M.; Harding, H. P.; Ron, D., Dynamic interaction of BiP and ER stress transducers in the unfolded-protein response. Nat Cell Biol 2000, 2, (6), 326-32.

14. Harding, H. P.; Ron, D., Endoplasmic reticulum stress and the development of diabetes: a review. Diabetes 2002, 51 Suppl 3 , S455-61.

15. Harding, H. P.; Novoa, I.; Zhang, Y.; Zeng, H.; Wek, R.; Schapira, M.; Ron, D., Regulated translation initiation controls stress-induced gene expression in mammalian cells. Mol Cell 2000, 6, (5), 1099-108.

16. He, C. H.; Gong, P.; Hu, B.; Stewart, D.; Choi, M. E.; Choi, A. M.; Alam, J., Identification of activating transcription factor 4 (ATF4) as an Nrf2-interacting protein. Implication for heme oxygenase-1 gene regulation. J Biol Chem 2001, 276, (24), 20858-65.

17. Choe, K. P.; Leung, C. K., SKN-1/Nrf, a new unfolded protein response factor? PLoS Genet 2013, 9, (9), e1003827.

18. Glover-Cutter, K. M.; Lin, S.; Blackwell, T. K., Integration of the unfolded protein and oxidative stress responses through SKN-1/Nrf. PLoS Genet 2013, 9, (9), e1003701.

19. Zhang, Y.; Kobayashi, A.; Yamamoto, M.; Hayes, J. D., The Nrf3 transcription factor is a membrane-bound glycoprotein targeted to the endoplasmic reticulum through its N-terminal homology box 1 sequence. J Biol Chem 2009, 284, (5), 3195-210.

20. Zhang, Y.; Lucocq, J. M.; Hayes, J. D., The Nrf1 CNC/bZIP protein is a nuclear envelope-bound transcription factor that is activated by t-butyl hydroquinone but not by endoplasmic reticulum stressors. Biochem J 2009, 418, (2), 293-310.

21. Zhao, R.; Hou, Y.; Xue, P.; Woods, C. G.; Fu, J.; Feng, B.; Guan, D.; Sun, G.; Chan, J. Y.; Waalkes, M. P.; Andersen, M. E.; Pi, J., Long isoforms of NRF1 contribute to arsenic-induced antioxidant response in human keratinocytes. Environ Health Perspect 2011, 119, (1), 56-62.

22. Sha, Z.; Goldberg, A. L., Proteasome-mediated processing of Nrf1 is essential for coordinate induction of all proteasome subunits and p97. Curr Biol 2014, 24, (14), 1573-1583.

23. Zhang, Y.; Nicholatos, J.; Dreier, J. R.; Ricoult, S. J.; Widenmaier, S. B.; Hotamisligil, G. S.; Kwiatkowski, D. J.; Manning, B. D., Coordinated regulation of protein synthesis and degradation by mTORC1. Nature 2014, 513, (7518), 440-3.

24. Xiang, Y.; Wang, M.; Hu, S.; Qiu, L.; Yang, F.; Zhang, Z.; Yu, S.; Pi, J.; Zhang, Y., Mechanisms controlling the multistage post-translational processing of endogenous Nrf1alpha/TCF11 proteins to yield distinct isoforms within the coupled positive and negative feedback circuits. Toxicol Appl Pharmacol 2018, 360, 212-235.

25. Ye, J.; Rawson, R. B.; Komuro, R.; Chen, X.; Dave, U. P.; Prywes, R.; Brown, M. S.; Goldstein, J. L., ER stress induces cleavage of membrane-bound ATF6 by the same proteases that process SREBPs. Mol Cell 2000, 6, (6), 1355-64.

26. Wang, Q.; Mora-Jensen, H.; Weniger, M. A.; Perez-Galan, P.; Wolford, C.; Hai, T.; Ron, D.; Chen, W.; Trenkle, W.; Wiestner, A.; Ye, Y., ERAD inhibitors integrate ER stress with an epigenetic mechanism to activate BH3-only protein NOXA in cancer cells. Proc Natl Acad Sci U S A 2009, 106, (7), 2200-5.

27. Lee, C. S.; Ho, D. V.; Chan, J. Y., Nuclear factor-erythroid 2-related factor 1 regulates expression of proteasome genes in hepatocytes and protects against endoplasmic reticulum stress and steatosis in mice. Febs j 2013, 280, (15), 3609-20.

28. Yuan, J.; Zhang, S.; Zhang, Y., Nrf1 is paved as a new strategic avenue to prevent and treat cancer, neurodegenerative and other diseases. Toxicol Appl Pharmacol 2018, 360, 273-283.

29. Steffen, J.; Seeger, M.; Koch, A.; Kruger, E., Proteasomal degradation is transcriptionally controlled by TCF11 via an ERAD-dependent feedback loop. Mol Cell 2010, 40, (1), 147-58.

30. Meister, S.; Schubert, U.; Neubert, K.; Herrmann, K.; Burger, R.; Gramatzki, M.; Hahn, S.; Schreiber, S.; Wilhelm, S.; Herrmann, M.; Jack, H. M.; Voll, R. E., Extensive immunoglobulin production sensitizes myeloma cells for proteasome inhibition. Cancer Res 2007, 67, (4), 1783-92.

31. Qiu, L.; Wang, M.; Hu, S.; Ru, X.; Ren, Y.; Zhang, Z.; Yu, S.; Zhang, Y., Oncogenic activation of Nrf2, though as a master antioxidant transcription factor, liberated by specific knockout of the full-length Nrflalpha that acts as a dominant tumor repressor. Cancers (Basel) 2018, 10, (12).

32. Xiang, Y.; Halin, J.; Fan, Z.; Hu, S.; Wang, M.; Qiu, L.; Zhang, Z.; Mattjus, P.; Zhang, Y., Topovectorial mechanisms control the juxtamembrane proteolytic processing of Nrf1 to remove its N-terminal polypeptides during maturation of the CNC-bZIP factor. Toxicol Appl Pharmacol 2018, 360, 160-184.

33. Zhang, Y.; Hayes, J. D., The membrane-topogenic vectorial behaviour of Nrf1 controls its post-translational modification and transactivation activity. Sci Rep 2013, 3, 2006.

34. Xu, Z.; Chen, L.; Leung, L.; Yen, T. S.; Lee, C.; Chan, J. Y., Liver-specific inactivation of the Nrf1 gene in adult mouse leads to nonalcoholic steatohepatitis and hepatic neoplasia. Proc Natl Acad Sci U S A 2005, 102, (11), 4120-5.

35. Pearse, B. R.; Hebert, D. N., Cotranslocational degradation: utilitarianism in the ER stress response. Mol Cell 2006, 23, (6), $773-5$. 
bioRxiv preprint doi: https://doi.org/10.1101/655159; this version posted May 31, 2019. The copyright holder for this preprint (which was not certified by peer review) is the author/funder. All rights reserved. No reuse allowed without permission.

36. Schroder, M.; Kaufman, R. J., The mammalian unfolded protein response. Annu Rev Biochem 2005, 74, 739-89.

37. Wouters, B. G.; Koritzinsky, M., Hypoxia signalling through mTOR and the unfolded protein response in cancer. Nat Rev Cancer 2008, 8, (11), 851-64.

38. Scriven, P.; Brown, N. J.; Pockley, A. G.; Wyld, L., The unfolded protein response and cancer: a brighter future unfolding? $J$ Mol Med (Berl) 2007, 85, (4), 331-41.

39. Hotamisligil, G. S., Endoplasmic reticulum stress and the inflammatory basis of metabolic disease. Cell 2010, 140, (6), 900-17.

40. Choi, H.; Jackson, N. L.; Shaw, D. R.; Emanuel, P. D.; Liu, Y. L.; Tousson, A.; Meng, Z.; Blume, S. W., mrtl-A translation/localization regulatory protein encoded within the human c-myc locus and distributed throughout the endoplasmic and nucleoplasmic reticular network. J Cell Biochem 2008, 105, (4), 1092-108.

41. Wethmar, K.; Smink, J. J.; Leutz, A., Upstream open reading frames: molecular switches in (patho)physiology. Bioessays 2010, $32,(10), 885-93$

42. Mielnicki, L. M.; Hughes, R. G.; Chevray, P. M.; Pruitt, S. C., Mutated Atf4 suppresses c-Ha-ras oncogene transcript levels and cellular transformation in NIH3T3 fibroblasts. Biochem Biophys Res Commun 1996, 228, (2), 586-95.

43. Tomlin, F. M.; Gerling-Driessen, U. I. M.; Liu, Y. C.; Flynn, R. A.; Vangala, J. R.; Lentz, C. S.; Clauder-Muenster, S.; Jakob, P.; Mueller, W. F.; Ordonez-Rueda, D.; Paulsen, M.; Matsui, N.; Foley, D.; Rafalko, A.; Suzuki, T.; Bogyo, M.; Steinmetz, L. M.;

Radhakrishnan, S. K.; Bertozzi, C. R., Inhibition of NGLY1 inactivates the transcription factor Nrf1 and potentiates proteasome inhibitor cytotoxicity. ACS Cent Sci 2017, 3, (11), 1143-1155.

44. Yang, K.; Huang, R.; Fujihira, H.; Suzuki, T.; Yan, N., N-glycanase NGLY1 regulates mitochondrial homeostasis and inflammation through NRF1. J Exp Med 2018, 215, (10), 2600-2616.

45. van Keulen, B. J.; Rotteveel, J.; Finken, M. J. J., Unexplained death in patients with NGLY1 mutations may be explained by adrenal insufficiency. Physiol Rep 2019, 7, (3), e13979.

46. Need, A. C.; Shashi, V.; Hitomi, Y.; Schoch, K.; Shianna, K. V.; McDonald, M. T.; Meisler, M. H.; Goldstein, D. B., Clinical application of exome sequencing in undiagnosed genetic conditions. J Med Genet 2012, 49, (6), 353-61.

47. Owings, K. G.; Lowry, J. B.; Bi, Y.; Might, M.; Chow, C. Y., Transcriptome and functional analysis in a Drosophila model of NGLY1 deficiency provides insight into therapeutic approaches. Hum Mol Genet 2018, 27, (6), 1055-1066.

48. Lehrbach, N. J.; Ruvkun, G., Endoplasmic reticulum-associated SKN-1A/Nrf1 mediates a cytoplasmic unfolded protein response and promotes longevity. eLife 2019, 8, e44425.

49. Lehrbach, N. J.; Breen, P. C.; Ruvkun, G., Protein sequence editing of SKN-1A/Nrf1 by peptide:N-Glycanase controls proteasome gene expression. Cell 2019, 177, (3), 737-750.e15.

50. Zhang, Y.; Lucocq, J. M.; Yamamoto, M.; Hayes, J. D., The NHB1 (N-terminal homology box 1) sequence in transcription factor $\mathrm{Nrf1}$ is required to anchor it to the endoplasmic reticulum and also to enable its asparagine-glycosylation. Biochem $J$ 2007, 408, (2), 161-72.

51. Zhang, Y.; Ren, Y.; Li, S.; Hayes, J. D., Transcription factor Nrf1 is topologically repartitioned across membranes to enable target gene transactivation through its acidic glucose-responsive domains. PLoS One 2014, 9, (4), e93458.

52. Zhang, Y.; Hayes, J. D., Identification of topological determinants in the N-terminal domain of transcription factor Nrf1 that control its orientation in the endoplasmic reticulum membrane. Biochem J 2010, 430, (3), 497-510.

53. Widenmaier, S. B.; Snyder, N. A.; Nguyen, T. B.; Arduini, A.; Lee, G. Y.; Arruda, A. P.; Saksi, J.; Bartelt, A.; Hotamisligil, G. S., NRF1 is an ER membrane sensor that is central to cholesterol homeostasis. Cell 2017, 171, (5), 1094-1109.e15.

54. Wang, X.; Sato, R.; Brown, M. S.; Hua, X.; Goldstein, J. L., SREBP-1, a membrane-bound transcription factor released by sterol-regulated proteolysis. Cell 1994, 77, (1), 53-62.

55. Sakai, J.; Duncan, E. A.; Rawson, R. B.; Hua, X.; Brown, M. S.; Goldstein, J. L., Sterol-regulated release of SREBP-2 from cell membranes requires two sequential cleavages, one within a transmembrane segment. Cell 1996, 85, (7), 1037-46.

56. Brown, M. S.; Goldstein, J. L., The SREBP pathway: regulation of cholesterol metabolism by proteolysis of a membrane-bound transcription factor. Cell 1997, 89, (3), 331-40.

57. Bogdanov, M.; Vitrac, H.; Dowhan, W., Flip-Flopping Membrane Proteins: How the Charge Balance Rule Governs Dynamic Membrane Protein Topology. In 2018; pp 1-28.

58. Zhang, Z.; Zhang, L.; Zhou, L.; Lei, Y.; Zhang, Y.; Huang, C., Redox signaling and unfolded protein response coordinate cell fate decisions under ER stress. Redox Biol 2018.

59. Ren, Y.; Qiu, L.; Lu, F.; Ru, X.; Li, S.; Xiang, Y.; Yu, S.; Zhang, Y., TALENs-directed knockout of the full-length transcription factor Nrf1alpha that represses malignant behaviour of human hepatocellular carcinoma (HepG2) cells. Sci Rep 2016, 6, 23775.

60. Wang, M.; Qiu, L.; Ru, X.; Song, Y.; Zhang, Y., Distinct isoforms of Nrf1 diversely regulate different subsets of its cognate target genes. Sci Rep 2019, 9, (1), 2960. 